\title{
The Steklov eigenvalue problem in a cuspidal domain
}

\author{
María G. Armentano and Ariel L. Lombardi
}

Received: date / Accepted: date

\begin{abstract}
In this paper we analyze the approximation, by piecewise linear finite elements, of a Steklov eigenvalue problem in a plane domain with an external cusp. This problem is not covered by the literature and its analysis requires a special treatment. Indeed, we develop new trace theorems and we also obtain regularity results for the source counterpart. Moreover, under appropriate assumptions on the meshes, we present interpolation error estimates for functions in fractional Sobolev spaces. These estimates allow us to obtain appropriate convergence results of the source counterpart which, in the context of the theory of compact operator, are a fundamental tool in order to prove the convergence of the eigenpairs. At the end, we prove the convergence of the eigenpairs by using graded meshes and present some numerical tests.
\end{abstract}

Keywords Steklov eigenvalue problem · finite elements · Cuspidal domains · Graded meshes

Mathematics Subject Classification (2000) 65N25, 65N30

\section{Introduction}

Problems with an eigenvalue parameter on the boundary, as the Steklov eigenvalue problem, arise in the study of many interesting physical situations. We refer to the survey article [16] where several applications and features of the Steklov spectrum are discussed. One of them is its essential role for the implementation of electrical impedance tomography in medical and geophysical imaging, and the analysis of photonic crystal. If $\Omega$ is two-dimensional, the Steklov eigenvalues can be thought as the squares of the natural frequencies of a vibrating free membrane with its mass concentrated along its boundary. This problem was also considered in [19], for modeling the sloshing problem which describes the natural frequencies and the corresponding modes of a free wave motion in an infinitely long canal having an uniform cross section. The Steklov eigenvalue problem under consideration arises, for example, in the study of the normal modes of oscillation of a mass of liquid in a finite basin. In this case the eigenvalue equation is satisfied only on the top of the boundary (the free surface), while on the bottom the vanishing of the normal derivative is required (see, for example, [14]). There are features of the Steklov eigenvalues which are, in comparison with the Laplacian eigenvalue problem with Dirichlet or Neumann conditions, highly

M. G. Armentano

Departamento de Matemática, Facultad de Ciencias Exactas y Naturales, Universidad de Buenos Aires, IMAS - CONICET, 1428 Buenos Aires, Argentina. E-mail: garmenta@dm.uba.ar

A. L. Lombardi

Departamento de Matemática, Facultad de Ciencias Exactas, Ingeniería y Agrimensura, Universidad Nacional de Rosario, Av. Pellegrini 250, 2000 Rosario, Argentina.

CONICET, Argentina. E-mail: ariel@fceia.unr.edu.ar 
sensible to the regularity of the boundary, as is explained in [16]. Indeed, even for Lipschitz domains, one-term spectral asymptotics have not been proved yet (see [16] and references therein).

Numerical approximations of eigenvalues and eigenfunctions, by the finite element method, have been widely analyzed for several kinds of spectral problems, and, in particular, for Steklov eigenvalue problems (see, for example, $[2,6,7,9]$ and the reference therein). However, as far as we know, all of them only deal with polygonal or smooth domains.

The goal of this paper is the analysis of a piecewise linear finite element approximation of a Steklov eigenvalue problem in a rough plane domain, precisely a domain with an external cusp. Such a domain is curved and non-Lipschitz, and therefore the classical spectral theory (see $[10,12,23]$ ) cannot be directly applied and special treatment is required. In order to be able to use part of the theory developed in [2-4] and with the aim to add new tools to it, we consider the model problem

$$
\left\{\begin{array}{rlrl}
-\Delta u & =0 & & \text { in } \Omega, \\
\frac{\partial u}{\partial \nu}=\lambda u & & \text { on } \Gamma_{3}, \\
\frac{\partial u}{\partial \nu}=0 & & \text { on } \Gamma_{1}, \\
u=0 & & \text { on } \Gamma_{2},
\end{array}\right.
$$

where $\Omega \subset \mathbb{R}^{2}$ is the domain defined by

$$
\Omega=\left\{(x, y): 0<x<1,0<y<x^{\alpha}\right\},
$$

$\alpha$ is a parameter satisfying $1<\alpha<2, \nu$ denotes the unitary outward normal to $\Omega$ (see Figure 1). With $\Gamma=\Gamma_{1} \cup \Gamma_{2} \cup \Gamma_{3}$ we denote the boundary of $\Omega$, where

$$
\Gamma_{1}=\{0 \leq x \leq 1, y=0\}, \quad \Gamma_{2}=\{x=1,0 \leq y \leq 1\} \text { and } \Gamma_{3}=\left\{0 \leq x \leq 1, y=x^{\alpha}\right\} .
$$

In view of the boundary conditions in the Steklov eigenvalue problem, a different analysis than the one developed in [2] for the Laplacian eigenvalue problem in this kind of domain, is required. Therefore, the results of the previous works in domains with an external cusp (see, [1-4] and the references therein) need to be extended or generalized. In particular, in this work we prove that functions in $H^{1}(\Omega)$ have traces in fractional Sobolev spaces, extending the trace theorems of [3]. We also analyze the existence of solutions for the source counterpart, to cover the case in which the boundary datum is less regular, extending the regularity results given in [3]. Moreover, we need piecewise linear interpolation error estimates for functions in fractional Sobolev spaces strictly between $H^{1}$ and $H^{2}$, which are obtained by using the theory of interpolation of operators. Since the Lagrange interpolation, used in $[2,4]$, is not defined for functions in $H^{1}$, we introduce a Scott-Zhang type quasi-interpolation and obtain stability and error estimates under appropriate conditions of the family of anisotropic meshes. It is important to emphasize that, due to the characteristics of the domain under consideration, these interpolation error estimates are not covered by the literature and provide, in particular, a new contribution to the interpolation theory in cuspidal domains. All these results allow us to obtain suitable convergence results for the source counterpart and prove, by using the abstract approximation theory stated in $[10,12,23]$, the convergence of the eigenfunctions and the eigenvalues when suitable graded meshes are used.

Let $V=\left\{v \in H^{1}(\Omega):\left.v\right|_{\Gamma_{2}}=0\right\}$. The weak form of the problem is: Find $\lambda \in \mathbb{R}$ and $u \in V, u \neq 0$ satisfying

$$
\left\{\begin{aligned}
a(u, v) & =\lambda b(u, v) \quad \forall v \in V, \\
\|u\|_{L^{2}\left(\Gamma_{3}\right)} & =1
\end{aligned}\right.
$$

where $a(u, v)=\int_{\Omega} \nabla u \cdot \nabla v$, which is continuous and coercive on $V$, and $b(u, v)=\int_{\Gamma_{3}} u v$.

To approximate the solution of (1) we replace $\Omega$ with a polygonal domain $\Omega_{h}$ and use the standard linear finite element method. We will construct $\Omega_{h}$ in such a way that $\Omega \subset \Omega_{h}$ and the nodes on $\Gamma_{h}$, the boundary of $\Omega_{h}$, are on $\Gamma$. 


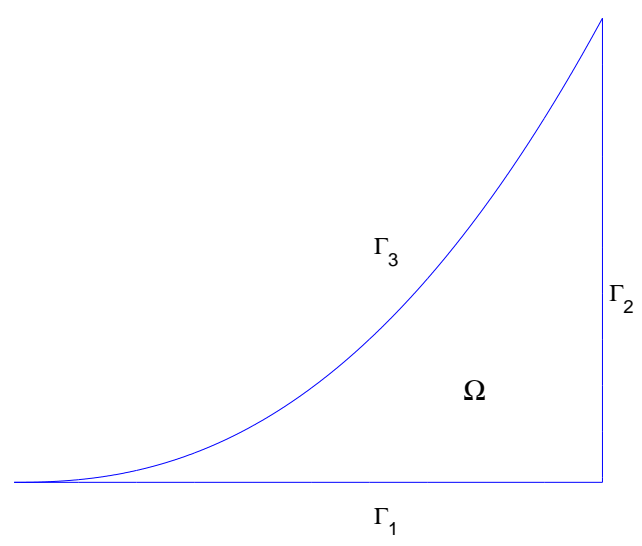

Fig. 1 A cuspidal domain

Let $\left\{\mathcal{T}_{h}\right\}$ be a family of triangulations of $\Omega_{h}$. Associated with $\left\{\mathcal{T}_{h}\right\}$ we have the finite element space

$$
V_{h}=\left\{v \in H^{1}\left(\Omega_{h}\right):\left.v\right|_{\Gamma_{2}}=0 \text { and }\left.v\right|_{K} \in \mathcal{P}_{1} \quad \forall K \in \mathcal{T}_{h}\right\}
$$

where $\mathcal{P}_{1}$ denotes the space of linear polynomials.

Then, we propose the following discrete problem: find $\lambda_{h} \in \mathbb{R}$ and $u_{h} \in V_{h}, u_{h} \neq 0$ such that

$$
\left\{\begin{aligned}
a_{h}\left(u_{h}, v\right) & =\lambda_{h} b\left(u_{h}, v\right) \quad \forall v \in V_{h}, \\
\left\|u_{h}\right\|_{L^{2}\left(\Gamma_{3}\right)} & =1,
\end{aligned}\right.
$$

where $a_{h}\left(u_{h}, v\right)=\int_{\Omega_{h}} \nabla u_{h} \cdot \nabla v$. We note that, although our numerical scheme (2) conserves the continuous bilinear form $b$ of $(1)$ on the right hand side, it is non conforming, since the integral in the definition of $a_{h}$ is on $\Omega_{h}$.

Let $L_{\beta}^{2}\left(\Gamma_{3}\right)$ be the space defined as $L_{\beta}^{2}\left(\Gamma_{3}\right)=\left\{v:\left\|v x^{\beta}\right\|_{L^{2}\left(\Gamma_{3}\right)}<\infty\right\}$, with $\beta \in \mathbb{R}$ such that $\beta=0$ if $\alpha \leq 2$ (see, $[3,22]$ ) and $\beta>\frac{\alpha}{2}-1$ if $\alpha>2$. We consider the following problem associated with the Steklov eigenvalues of the Laplacian operator: Given $g \in L_{-\beta}^{2}\left(\Gamma_{3}\right)$, find $u \in V$ such that,

$$
a(u, v)=\int_{\Gamma_{3}} g v \quad \forall v \in V .
$$

From the trace theorems obtained in [3] we can affirm that, this problem has a unique solution $u \in V$.

In order to analyze the convergence properties of (2) we use the abstract approximation theory stated in $[10,12,23]$. Therefore we need to introduce appropriate compact operators $T$ and $T_{h}$ associated to (1) and (2) respectively defined in the same functional space, a requirement not easy to fulfill, and such that $T_{h} \rightarrow T$ in norm. Due to the Neumann boundary conditions in the Steklov eigenvalue problem, we need to develop new trace theorems which in particular allow us to prove the compactness of the involved operators. We also show the regularity of the solution of the source counterpart in fractional Sobolev spaces. Then, in order to prove the convergence of $T_{h}$ to $T$, we obtain interpolation error estimates in fractional Sobolev spaces taking into account that our meshes necessarily include anisotropic elements, such as those with a vertex at the tip of the cusp, and therefore these estimates are not standard and require a special analysis.

The rest of the paper is organized as follows. In Section 2 we state the spectral problem, so we introduce linear operators $T$ and $T_{h}$ associated to the eigenvalue problem and its approximation. We present a trace theorem, which is an important tool in order to prove the compactness of the operators, and we also obtain some regularity results for the solution of the associated source problems. In Section 3 we present the interpolation error estimates in fractional Sobolev spaces for some anisotropic graded meshes. In section 4 we obtain $L^{2}$ error estimates on $\Gamma_{3}$. In Section 5 , we prove the convergence of the 
eigenfunctions and eigenvalues. Finally, in section 6 we analyze numerical aspects concerning the solution of the discrete generalized eigenvalue problem (2) and we present some numerical examples.

\section{The spectral problems}

In this section we introduce appropriate linear operators $T$ and $T_{h}$ with spectra related to those of problems (1) and (2) respectively. Despite of $\alpha$ is a parameter satisfying $1<\alpha<2$, some results, as specified in the statements, are also valid for $\alpha=2$.

Let $B: L^{2}\left(\Gamma_{3}\right) \rightarrow H^{1}(\Omega)$ be the solution operator of problem (3) defined by $B g=u$. In view of Theorem 2.2 of [3] and, for the case $\alpha=2$, Lemma 2.1 of [22], we can define the operator $T: L^{2}\left(\Gamma_{3}\right) \rightarrow$ $L^{2}\left(\Gamma_{3}\right)$ in such a way that for $g \in L^{2}\left(\Gamma_{3}\right), T g$ is the restriction of $B g$ on $\Gamma_{3}: T g=\left.B g\right|_{\Gamma_{3}}$. In this case we have that

$$
\|T g\|_{L^{2}\left(\Gamma_{3}\right)} \leq C\|u\|_{H^{1}(\Omega)} \leq C\|g\|_{L^{2}\left(\Gamma_{3}\right)} .
$$

This inequality guarantee that the operator $T$ is bounded in $L^{2}\left(\Gamma_{3}\right)$.

Hence, we want to obtain conditions under which the operator $T$ is compact. In the case of a Lipschitz domain, the compactness of this kind of operator follows by using well known results on restrictions of $H^{1}(\Omega)$ to the boundary (see, for example, [6]) but, since our domain is not Lipschitz, those arguments can not be straightforward applied.

For any subset $D \subset \mathbb{R}^{2}$ and $0<s<1$, we recall that the space $H^{s}(D)$ is defined as

$$
H^{s}(D)=\left\{v \in L^{2}(D):\|v\|_{H^{s}(D)}<\infty\right\}
$$

with

$$
\|v\|_{H^{s}(D)}=\|v\|_{L^{2}(D)}+|v|_{H^{s}(D)}
$$

and

$$
|v|_{H^{s}(D)}=\left(\int_{D} \int_{D} \frac{\mid v(\mathbf{x})-v(\mathbf{y}))\left.\right|^{2}}{\|\mathbf{x}-\mathbf{y}\|^{1+2 s}} d \mathbf{x} d \mathbf{y}\right)^{\frac{1}{2}} .
$$

Then, the following example shows that although the restriction of $H^{1}(\Omega)$ functions is in $L^{2}(\Gamma)$ for $\alpha \leq 2$, it is not necessarily in $H^{s}(\Gamma)$ for $s>1-\frac{\alpha}{2}$, and so, the trace may be not in $H^{\frac{1}{2}}(\Gamma)$.

Example 1 Let $v$ be the function $v(x, y)=x^{\gamma}$ with $\gamma<0$. Then, an easy computation shows that $v \in H^{1}(\Omega)$ iff $\gamma>\frac{1-\alpha}{2}$ and $v \in L^{2}(\Gamma)$ iff $\gamma>-\frac{1}{2}$. So, for $\alpha<2$ and $\gamma>\frac{1-\alpha}{2}$ this function is in $H^{1}(\Omega)$ and in $L^{2}(\Gamma)$. However, we show that $v$ is not in $H^{s}(\Gamma)$ if $\gamma \leq s-\frac{1}{2}$ and therefore, for $\alpha<2$ there exist functions which are in $H^{1}(\Omega)$ and $L^{2}(\Gamma)$ but not in $H^{s}(\Gamma)$ for $s$ with $1>s>1-\frac{\alpha}{2}$ (observe that $1-\frac{\alpha}{2} \nearrow \frac{1}{2}$ when $\alpha \searrow 1$, and $1-\frac{\alpha}{2} \searrow 0$ when $\left.\alpha \nearrow 2\right)$.

In fact,

$$
\begin{aligned}
\int_{\Gamma_{1}} \int_{\Gamma_{1}} \frac{\mid v(\mathbf{x})-v(\mathbf{y}))\left.\right|^{2}}{\|\mathbf{x}-\mathbf{y}\|^{1+2 s}} d \mathbf{x} d \mathbf{y} & =\int_{0}^{1} \int_{0}^{y} \frac{\left|y^{\gamma}-x^{\gamma}\right|^{2}}{(y-x)^{1+2 s}} d x d y+\int_{0}^{1} \int_{y}^{1} \frac{\left|x^{\gamma}-y^{\gamma}\right|^{2}}{(x-y)^{1+2 s}} d x d y \\
& =I+I I .
\end{aligned}
$$

Now, since $s<1$ and by introducing $\theta_{x, y}$ with $x<\theta_{x, y}<y$ and defined by $x^{\gamma}-y^{\gamma}=\gamma \theta_{x, y}^{\gamma-1}(x-y)$, we have

$$
\begin{aligned}
I & =\int_{0}^{1} \int_{0}^{y} \frac{\gamma^{2} \theta_{x, y}^{2 \gamma-2}(y-x)^{2}}{(y-x)^{1+2 s}} d x d y \geq \int_{0}^{1} \int_{0}^{y} \frac{\gamma^{2} y^{2 \gamma-2}}{(y-x)^{2 s-1}} d x d y \\
& =\int_{0}^{1} \frac{\gamma^{2}}{2-2 s} y^{2 \gamma-2} y^{2-2 s} d y=\frac{\gamma^{2}}{2-2 s} \int_{0}^{1} y^{2 \gamma-2 s} d y .
\end{aligned}
$$


Then, taking into account that $I I \geq 0$, we get

$$
|v|_{H^{s}\left(\Gamma_{1}\right)}=I+I I \geq \frac{\gamma^{2}}{2-2 s} \int_{0}^{1} y^{2 \gamma-2 s} d y .
$$

Therefore, if $\gamma \leq s-\frac{1}{2}$, the function $v \notin H^{s}\left(\Gamma_{1}\right)$ (and the same result holds on $\Gamma_{3}$ since $\left|\left(1, \alpha x^{\alpha-1}\right)\right|$ is bounded). Moreover, since $\|v\|_{H^{s}\left(\Gamma_{i}\right)}<\|v\|_{H^{s}(\Gamma)}, i=1,2,3$, we can conclude that $v \notin H^{s}(\Gamma)$.

Next theorem allows us to prove that, for $1<\alpha \leq 2$, the restriction of $H^{1}(\Omega)$ functions are in $H^{\sigma}(\Gamma)$, with $\sigma=1-\frac{\alpha}{2}$.

Theorem 1 Let $1<\alpha<2$. There exists a constant $C$, depending only on $\alpha$, such that for any $u \in H^{1}(\Omega)$

$$
\|u\|_{H^{\sigma}\left(\Gamma_{i}\right)} \leq C\|u\|_{H^{1}(\Omega)}
$$

with $\sigma=1-\frac{\alpha}{2}$ and $i=1,2,3$.

Proof. Let $\mathcal{D}=\left\{(x, y):-x<y<x^{\alpha}, 0<x<1\right\}$ and $\tilde{u}: \mathcal{D} \rightarrow \mathbb{R}$ be the extension of $u$ to $\mathcal{D}$ defined in [4]. By examining the proof of Lemma 3.1 of [4] we know that $\tilde{u} \in H^{1}\left(\mathcal{D}, r^{\alpha-1}\right)$ and

$$
\|\tilde{u}\|_{H^{1}\left(\mathcal{D}, r^{\alpha-1}\right)} \leq 2\|u\|_{H^{1}(\Omega)},
$$

where $r=\sqrt{x^{2}+y^{2}}$ and, for any function $\rho \geq 0$ and $s \in \mathbb{R}$, we define

$$
\|v\|_{H^{1}\left(\mathcal{D}, \rho^{s}\right)}^{2}:=\left\|v \rho^{\frac{s}{2}}\right\|_{L^{2}(\mathcal{D})}^{2}+\left\|\nabla v \rho^{\frac{s}{2}}\right\|_{L^{2}(\mathcal{D})}^{2} .
$$

We observe that, if $d(x, y)$ denotes the distance between $(x, y)$ and $\partial \mathcal{D}$ then, $d(x, y) \leq r(x, y)$ for any $(x, y) \in \mathcal{D}$. Thus,

$$
\tilde{u} \in H^{1}\left(\mathcal{D}, d^{\alpha-1}\right), \quad\|\tilde{u}\|_{H^{1}\left(\mathcal{D}, d^{\alpha-1}\right)} \leq C\|u\|_{H^{1}(\Omega)} .
$$

Now, since $\mathcal{D}$ is Lipschitz we can apply [18, Theorem 2.13] (taking $p=2, d=2, k=1, \theta=\alpha+1$ and $\sigma=1-\frac{\alpha}{2}$ ), or [13, Theorem 3.2] (with $p=2$ and $a=\frac{\alpha-1}{2}$ ), to conclude that for a constant $C$, depending only on $\alpha$, we have

$$
\|\tilde{u}\|_{H^{\sigma}(\partial \mathcal{D})} \leq C\|\tilde{u}\|_{H^{1}\left(\mathcal{D}, d^{\alpha-1}\right)} \leq C\|u\|_{H^{1}(\Omega)} .
$$

In particular, for $i=2,3$ we have that

$$
\|u\|_{H^{\sigma}\left(\Gamma_{i}\right)} \leq\|\tilde{u}\|_{H^{\sigma}(\Gamma)} \leq C\|u\|_{H^{1}(\Omega)} .
$$

The estimate for $i=1$ follows by similar arguments.

Corollary 1 There exists a constant $C$, depending only on $\alpha$, such that for any $u \in H^{1}(\Omega)$ we have

$$
\|u\|_{H^{\sigma}(\Gamma)} \leq C\|u\|_{H^{1}(\Omega)}
$$

with $\sigma=1-\frac{\alpha}{2}, 1<\alpha<2$.

Proof. From Theorem 1 we know that, for $i=1,2,3, \gamma_{i}=\left.u\right|_{\Gamma_{i}}$ is in $H^{\sigma}\left(\Gamma_{i}\right)$ with $\sigma=1-\frac{\alpha}{2}<\frac{1}{2}$. Since $\sigma<\frac{1}{2}$, if we consider $\tilde{\gamma}_{i}$ the extension by zero of $\gamma_{i}$ to $\Gamma$ we can affirm that $\tilde{\gamma}_{i}$ is in $H^{\sigma}(\Gamma)[20$, Theorem 11.4]. Then

$$
\left\|\tilde{\gamma}_{i}\right\|_{H^{\sigma}(\Gamma)}=C\left\|\tilde{\gamma}_{i}\right\|_{H^{\sigma}\left(\Gamma_{i}\right)} \leq C\|u\|_{H^{1}(\Omega)} .
$$

Hence, $\left.u\right|_{\Gamma}=\tilde{\gamma}_{1}+\tilde{\gamma}_{2}+\tilde{\gamma}_{3}$, and

$$
\|u\|_{H^{\sigma}(\Gamma)}=\left\|\tilde{\gamma}_{1}+\tilde{\gamma}_{2}+\tilde{\gamma}_{3}\right\|_{H^{\sigma}(\Gamma)} \leq\left\|\tilde{\gamma}_{1}\right\|_{H^{\sigma}(\Gamma)}+\left\|\tilde{\gamma}_{2}\right\|_{H^{\sigma}(\Gamma)}+\left\|\tilde{\gamma}_{3}\right\|_{H^{\sigma}(\Gamma)} \leq C\|u\|_{H^{1}(\Omega)},
$$

which concludes the proof. 
Next theorem (which is a consequence of Theorem 3.1 of [3]) guarantees the regularity of our source problem under appropriate conditions on the function $g$.

Proposition 1 Let $1<\alpha \leq 2$ and $g$ such that, if $h(t)=g\left(t, t^{\alpha}\right), h$ is in $H^{1}(0,1)$ and $h(0)=0$. Then, the solution $u$ of the source problem (3) is in $H^{2}(\Omega)$ and there exists a constant $C$ such that

$$
\|u\|_{H^{2}(\Omega)} \leq C\left\|h^{\prime}\right\|_{L^{2}(0,1)}
$$

Proof. We note that if $\varphi(t)=t^{\alpha}$ then

1. $\left\|h(t) \varphi^{-\frac{1}{2}}\right\|_{L^{2}(0,1)}=\left\|h(t) t^{-\frac{\alpha}{2}}\right\|_{L^{2}(0,1)} \leq C\left\|h^{\prime}\right\|_{L^{2}(0,1)}$, using the classical Hardy inequality and taking into account that $1<\alpha \leq 2$,

2. $\left(\varphi^{\prime \prime}\right)^{-\frac{1}{2}} \in L^{\infty}([0,1])$,

3. $\frac{\varphi^{\prime \prime} \varphi}{\left(\varphi^{\prime}\right)^{2}}$ is constant.

So the desired result follows by using Theorem 3.1 of [3] with $f=0$.

Given a Sobolev space $W\left(\Gamma_{3}\right)$, we denote by $\stackrel{o}{W}\left(\Gamma_{3}\right)$ the space defined as

$$
\stackrel{o}{W}\left(\Gamma_{3}\right)=\overline{\left\{\phi \in C^{\infty}\left(\Gamma_{3}\right): \phi(0,0)=0\right\}} \|^{\|\cdot\|_{W\left(\Gamma_{3}\right)}} .
$$

From [3, Theorem 2.2] and Proposition 1 we know that, if $g \in L^{2}\left(\Gamma_{3}\right)=\stackrel{o}{L^{2}}\left(\Gamma_{3}\right)$ the solution of $(3)$ is in $H^{1}(\Omega)$ and if $g \in \stackrel{o}{H^{1}}\left(\Gamma_{3}\right)$ then the solution of $(3)$ is in $H^{2}(\Omega)$. Let $0<s<\frac{1}{2}$. Following the theory developed in [20, Section 11.5], we have

$$
\left[L^{2}\left(\Gamma_{3}\right), \stackrel{o}{H^{1}}\left(\Gamma_{3}\right)\right]_{s}=\stackrel{o}{H^{s}}\left(\Gamma_{3}\right)=H^{s}\left(\Gamma_{3}\right),
$$

by using, for example, Corollary 1.4.4.5 of [17], for the last equality. Then if $g \in H^{s}\left(\Gamma_{3}\right)$, it follows from the theory of Interpolation of Operators (see for example [20, Theorem 5.1]) that the solution $u$ verifies

$$
u \in\left[H^{1}(\Omega), H^{2}(\Omega)\right]_{s}=H^{1+s}(\Omega)
$$

and we have the following theorem.

Theorem 2 Let $g \in H^{s}\left(\Gamma_{3}\right)$ with $0 \leq s<\frac{1}{2}$. Then, the solution $u$ of (3) is in $H^{1+s}(\Omega)$ and there exists a constant $C$, independent of $s$ but might depend on $\alpha$, such that

$$
\|u\|_{H^{1+s}(\Omega)} \leq C\|g\|_{H^{s}\left(\Gamma_{3}\right)} .
$$

Proposition 2 Assume $1<\alpha<2$. Then the operator $T: L^{2}\left(\Gamma_{3}\right) \rightarrow L^{2}\left(\Gamma_{3}\right)$ is compact and self-adjoint.

Proof. Using Theorem 1 with $\sigma=1-\frac{\alpha}{2}>0$, we have that

$$
\|T g\|_{H^{\sigma}\left(\Gamma_{3}\right)}=\left\|\left.u\right|_{\Gamma_{3}}\right\|_{H^{\sigma}\left(\Gamma_{3}\right)} \leq C\|u\|_{H^{1}(\Omega)} \leq C\|g\|_{L^{2}\left(\Gamma_{3}\right)},
$$

for all $g \in L^{2}\left(\Gamma_{3}\right)$. Therefore, the compactness of $T$ follows from the fact that for $\sigma>0, H^{\sigma}\left(\Gamma_{3}\right)$ is compactly embedded in $L^{2}\left(\Gamma_{3}\right)$. Now, for $g_{1}, g_{2} \in L^{2}\left(\Gamma_{3}\right)$, consider $u_{1}=B g_{1}$ and $u_{2}=B g_{2}$. Then, recalling the definition of $B$ given at the beginning of this Section (i.e., the solution operator of problem (3)) we have

$$
\left(T g_{1}, g_{2}\right)_{\Gamma_{3}}=\left(u_{1}, g_{2}\right)_{\Gamma_{3}}=a\left(u_{2}, u_{1}\right)=a\left(u_{1}, u_{2}\right)=\left(g_{1}, u_{2}\right)_{\Gamma_{3}}=\left(g_{1}, T g_{2}\right)_{\Gamma_{3}},
$$

which proves that $T$ is self-adjoint. 
Now, if we denote by $\mu$ a nonzero, real and simple eigenvalue of $T$ and by $z \in L^{2}\left(\Gamma_{3}\right)$ the associated eigenfunction, i.e., $T z=\mu z$, we get

$$
a(B z, v)=\int_{\Gamma_{3}} z v=\frac{1}{\mu} \int_{\Gamma_{3}} T z v=\frac{1}{\mu} \int_{\Gamma_{3}} B z v
$$

and so $\frac{1}{\mu}$ is an eigenvalue of (1) with eigenfunction $B z$.

Through this correspondence, properties of the eigenvalue problem (1) can be derived from the spectral theory for compact operators (see, for example, $[6,7,10,26]$ ). Thus, we may infer that the problem (1) has a sequence of eigenpairs $\left(\lambda_{j}, u_{j}\right)$, with positive eigenvalues $\lambda_{j}$ diverging to $+\infty$. We assume the eigenvalues to be increasingly ordered: $0<\lambda_{1} \leq \lambda_{2} \leq \cdots \leq \lambda_{j} \leq \cdots$.

Now, we want to introduce the analogous discrete operator $T_{h}$. Hence, we can consider the discrete problem: Find $u_{h} \in V_{h}$ such that

$$
a_{h}\left(u_{h}, v_{h}\right)=b\left(g, v_{h}\right) \quad \forall v_{h} \in V_{h} .
$$

We can define the solution operator $B_{h}: L^{2}\left(\Gamma_{3}\right) \rightarrow H^{1}\left(\Omega_{h}\right)$ by $B_{h} g=u_{h}$. Then, we take $T_{h}:$ $L^{2}\left(\Gamma_{3}\right) \rightarrow L^{2}\left(\Gamma_{3}\right)$ as the restriction on $\Gamma_{3}$ of applying $B_{h}: T_{h} g=\left.B_{h} g\right|_{\Gamma_{3}}$ which satisfies

$$
\left\|T_{h} g\right\|_{L^{2}\left(\Gamma_{3}\right)} \leq C\left\|u_{h}\right\|_{H^{1}\left(\Omega_{h}\right)} \leq C\|g\|_{L^{2}\left(\Gamma_{3}\right)}
$$

We observe that, our formulation of the discrete problem, allows us to define $T_{h}$ in the same functional space as $T$. Now, if we denote by $\mu_{h}$ a nonzero and real eigenvalue of $T_{h}$ and by $z_{h} \in L^{2}\left(\Gamma_{3}\right)$ the associated eigenfunction, i.e., $T_{h} z_{h}=\mu_{h} z_{h}$, we get

$$
a_{h}\left(B_{h} z_{h}, v_{h}\right)=\int_{\Gamma_{3}} z_{h} v_{h}=\frac{1}{\mu_{h}} \int_{\Gamma_{3}} T_{h} z_{h} v_{h}=\frac{1}{\mu_{h}} \int_{\Gamma_{3}} B_{h} z_{h} v_{h},
$$

as a consequence $\frac{1}{\mu_{h}}$ is an eigenvalue of (2) with eigenfunction $B_{h} z_{h}$.

In order to prove the convergence of the eigenvalues we want to use the classical spectral theory. Our first goal is to prove the convergence of $T_{h}$ to $T$ in norm.

\section{Graded meshes and interpolation error estimates}

We will assume that the family of meshes $\left\{\mathcal{T}_{h}\right\}$ satisfies the same properties considered in $[1,2,4]$. Indeed, let $\mathcal{T}_{h}$ be a triangulation of $\Omega_{h}$, where $\Omega_{h}$ is a polygonal approximation of $\Omega$ with all its vertices belonging to $\Gamma$, and $h>0$ be a parameter that goes to 0 . Let be $\gamma=(\alpha-1) / 2$ with $1<\alpha<2$. For each $K \in \mathcal{T}_{h}$ we denote by $h_{K}$ its diameter and by $\theta_{K}$ its maximum angle, we assume that there exist positive constants $\tau$ and $\theta_{M}<\pi$, independent of $h$, such that

M.1 $\theta_{K}<\theta_{M}, \forall K \in \mathcal{T}_{h}$ (the maximum angle condition).

M.2 $h_{K} \sim \tau h^{\frac{1}{1-\gamma}}$, if $(0,0) \in K$.

M.3 $h_{K} \leq \tau h \inf _{K} x^{\gamma}$, if $(0,0) \notin K$.

In order to simplify the proofs we also assume

M.4 Every triangle in $\mathcal{T}_{h}$ has a vertical (parallel to the $y$-axis) side.

In what follows we use the letter $C$ to denote a generic constant which is independent of $h$.

We say that two elements $K$ and $K^{\prime}$ are neighbors if $\bar{K} \cap \bar{K}^{\prime} \neq \emptyset$. We need the next assumption concerning neighboring elements: 


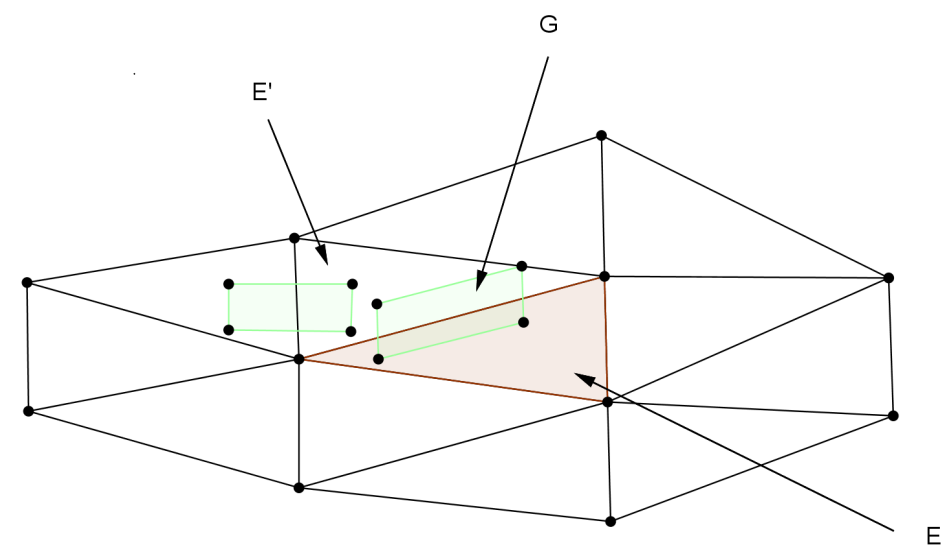

Fig. 2

M.5 Let $K$ and $K^{\prime}$ be neighbor elements, and suppose that $\ell_{1}, \ell_{2}$ are two different edges of $K$ and $\ell_{1}^{\prime}, \ell_{2}^{\prime}$ are different edges of $K^{\prime}$, being $\ell_{1}$ and $\ell_{1}^{\prime}$ vertical edges. Then we have

$$
\left|\ell_{1}\right| \leq C\left|\ell_{1}^{\prime}\right|, \quad\left|\ell_{2}\right| \leq C\left|\ell_{2}^{\prime}\right|
$$

with the constant $C$ independent of $K, K^{\prime}$ and $h$.

Assumption M.5 together with the maximum angle condition M.1 implies

$$
|K| \leq C\left|K^{\prime}\right|, \quad \forall K, K^{\prime} \in \mathcal{T}_{h} \text { such that } \bar{K} \cap \bar{K}^{\prime} \neq \emptyset
$$

We finally assume

M.6 For any element $K$ of $\mathcal{T}_{h}$ with vertices $\mathrm{n}_{1}, \mathrm{n}_{2}$ and $\mathrm{n}_{3}$, we denote by $\overline{\mathrm{n}_{i} \mathrm{n}_{j}}$ the edge with end points $\mathrm{n}_{i}$ and $\mathrm{n}_{j}, i, j=1,2,3$. Suppose that $\overline{\mathrm{n}_{2} \mathrm{n}_{3}}$ is the vertical edge of $K$. Then $\left|\overline{\mathrm{n}_{2} \mathrm{n}_{3}}\right| \leq C\left|\overline{\mathrm{n}_{1} \mathrm{n}_{2}}\right|$ and $\left|\overline{\mathrm{n}_{2} \mathrm{n}_{3}}\right| \leq C\left|\overline{\mathrm{n}_{1} \mathrm{n}_{3}}\right|$, with the constant $C$ independent of $K$ and $h$. Moreover, in the case that $\bar{K}$ intersects $\Gamma_{2}$ we also assume that $\left|\overline{\mathrm{n}_{1} \mathrm{n}_{2}}\right| \leq C\left|\overline{\mathrm{n}_{2} \mathrm{n}_{3}}\right|$ and $\left|\overline{\mathrm{n}_{1} \mathrm{n}_{3}}\right| \leq C\left|\overline{\mathrm{n}_{2} \mathrm{n}_{3}}\right|$.

We denote by $\mathcal{N}_{h}$ and $\mathcal{E}_{h}$ the sets of nodes and edges (element sides) of $\mathcal{T}_{h}$. Let $\mathcal{T}_{h}^{\Omega}$ be the partition of $\Omega$ defined by $\mathcal{T}_{h}$, that is

$$
\mathcal{T}_{h}^{\Omega}=\left\{E=K \cap \Omega: K \in \mathcal{T}_{h}\right\} .
$$

Note that an element in $\mathcal{T}_{h}^{\Omega}$ is either an element of $\mathcal{T}_{h}$ or a curved element with the curved edge on $\Gamma_{3}$. From now on, the word element refers to curved or non-curved elements. For each node $\mathrm{n}$, let $\omega_{\mathrm{n}}=\cup\left\{E \in \mathcal{T}_{h}^{\Omega}: \mathrm{n} \in \bar{E}\right\}$, and for an edge $\ell$ let $\omega_{\ell}=\omega_{\mathrm{n}} \cup \omega_{\mathrm{n}^{\prime}}$ if $\mathrm{n}$ and $\mathrm{n}^{\prime}$ are the end points of $\ell$. We put $\omega_{E}=\cup\left\{\omega_{\ell}: \ell\right.$ edge of $\left.E\right\}$ and finally $\omega_{E}^{\prime}$ is the subset of $\omega_{E}$ obtained by excluding curved elements.

The goal of this section is to obtain interpolation error estimates in fractional Sobolev spaces. First, we present the following estimate that will be useful later on.

Proposition 3 Let $E$ be a (non curved) element of $\mathcal{T}_{h}^{\Omega}$. Then there exists a constant $C$, depending only on the maximum angle of $\mathcal{T}_{h}$, and on the number of neighbours of $E$ such that for all $v \in H^{1}\left(\omega_{E}\right)$ we have

$$
\left\|v-c_{E}\right\|_{L^{2}\left(\omega_{E}^{\prime}\right)} \leq C\left(h_{E, 1}\left\|\partial_{x} v\right\|_{L^{2}\left(\omega_{E}^{\prime}\right)}+h_{E, 2}\left\|\partial_{y} v\right\|_{L^{2}\left(\omega_{E}^{\prime}\right)}\right)
$$

where $h_{E, 2}$ is the length of the vertical side of $E, h_{E, 1}$ is the length of $E$ in the $x$-direction and $c_{E}$ is the mean value of $v$ on $E$. 
Proof. Although the proof follows the arguments of [15, Theorem 7.1], we include it in detail with the purpose of showing that the interpolation constant depends only on the maximum angle of the mesh. Let $E^{\prime}$ be an element in $\omega_{E}^{\prime}$ sharing an edge with $E$. We first prove the inequality in $E \cup E^{\prime}$. Let $G$ be a parallelogram with

$$
|G \cap E| \sim|E|, \quad\left|G \cap E^{\prime}\right| \sim|E|,
$$

see Figure 2. The existence of such a $G$ follows from the assumptions on the mesh $\mathcal{T}_{h}$. Given $v \in H^{1}\left(\omega_{E}\right)$ we denote by $c_{S}$ the mean of $v$ on the set $S$. We know that

$$
\begin{aligned}
\left\|v-c_{E}\right\|_{L^{2}(E)} & \leq C\left(h_{E, 1}\left\|\partial_{x} v\right\|_{L^{2}(E)}+h_{E, 2}\left\|\partial_{y} v\right\|_{L^{2}(E)}\right), \\
\left\|v-c_{E^{\prime}}\right\|_{L^{2}\left(E^{\prime}\right)} & \leq C\left(h_{E, 1}\left\|\partial_{x} v\right\|_{L^{2}\left(E^{\prime}\right)}+h_{E, 2}\left\|\partial_{y} v\right\|_{L^{2}\left(E^{\prime}\right)}\right), \\
\left\|v-c_{G}\right\|_{L^{2}(G)} & \leq C\left(h_{E, 1}\left\|\partial_{x} v\right\|_{L^{2}(G)}+h_{E, 2}\left\|\partial_{y} v\right\|_{L^{2}(G)}\right)
\end{aligned}
$$

where we used that the diameters of $E, E^{\prime}$ and $G$ in the directions $x$ and $y$ are similar. Then

$$
\begin{aligned}
\left\|v-c_{E}\right\|_{L^{2}(E \cup G)} & \leq\left\|v-c_{E}\right\|_{L^{2}(E)}+\left\|v-c_{E}\right\|_{L^{2}(G)} \\
& \leq\left\|v-c_{E}\right\|_{L^{2}(E)}+\left\|v-c_{G}\right\|_{L^{2}(G)}+\left\|c_{G}-c_{E}\right\|_{L^{2}(G)} .
\end{aligned}
$$

From the equivalence (7) of measures and with $c_{G}$ and $c_{E}$ being constants we obtain

$$
\begin{aligned}
\left\|v-c_{E}\right\|_{L^{2}(E \cup G)} & \leq\left\|v-c_{E}\right\|_{L^{2}(E)}+\left\|v-c_{G}\right\|_{L^{2}(G)}+C\left\|c_{G}-c_{E}\right\|_{L^{2}(E \cap G)} \\
& \leq(C+1)\left(\left\|v-c_{E}\right\|_{L^{2}(E)}+\left\|v-c_{G}\right\|_{L^{2}(G)}\right) \\
& \leq C\left(h_{E, 1}\left\|\partial_{x} v\right\|_{L^{2}(E \cup G)}+h_{E, 2}\left\|\partial_{y} v\right\|_{L^{2}(E \cup G)}\right)
\end{aligned}
$$

where in the last line we used (8) and (10). Now, applying the same technique we have

$$
\begin{aligned}
\left\|v-c_{E}\right\|_{L^{2}\left(E \cup E^{\prime}\right)} & \leq\left\|v-c_{E}\right\|_{L^{2}(E \cup G)}+\left\|v-c_{E}\right\|_{L^{2}\left(E^{\prime}\right)} \\
& \leq\left\|v-c_{E}\right\|_{L^{2}(E \cup G)}+\left\|v-c_{E^{\prime}}\right\|_{L^{2}\left(E^{\prime}\right)}+\left\|c_{E}-c_{E^{\prime}}\right\|_{L^{2}\left(E^{\prime}\right)} \\
& \leq\left\|v-c_{E}\right\|_{L^{2}(E \cup G)}+\left\|v-c_{E^{\prime}}\right\|_{L^{2}\left(E^{\prime}\right)}+C\left\|c_{E^{\prime}}-c_{E}\right\|_{L^{2}\left(E^{\prime} \cap G\right)} \\
& \leq(C+1)\left(\left\|v-c_{E}\right\|_{L^{2}(E \cup G)}+\left\|v-c_{E^{\prime}}\right\|_{L^{2}\left(E^{\prime}\right)}\right) \\
& \leq C\left(h_{E, 1}\left\|\partial_{x} v\right\|_{L^{2}\left(E \cup E^{\prime}\right)}+h_{E, 2}\left\|\partial_{y} v\right\|_{L^{2}\left(E \cup E^{\prime}\right)}\right)
\end{aligned}
$$

where we used (11) and (9) in the last line. Repeating this argument for all the elements in $\omega_{E}^{\prime}$ we obtain the result.

Remark 1 Let $E_{0}$ be the element of $\mathcal{T}_{h}$ with $(0,0)$ as a vertex. Then, analogously to the proof of the last Proposition, we can obtain that there exist a constant $c_{E_{0}}$ such that

$$
\left\|v-c_{E_{0}}\right\|_{L^{2}\left(\omega_{E_{0}}^{\prime \prime}\right)} \leq C\left(h_{E_{0}, 1}\left\|\partial_{x} v\right\|_{L^{2}\left(\omega_{E_{0}}^{\prime \prime}\right)}+h_{E_{0}, 2}\left\|\partial_{y} v\right\|_{L^{2}\left(\omega_{E_{0}}^{\prime \prime}\right)}\right)
$$

where $\omega_{E_{0}}^{\prime \prime}=E_{0} \cup \omega_{E_{0}}^{\prime}$

Now we introduce a quasi-interpolation operator of Scott-Zhang type [25] $Q_{h}: H^{1}(\Omega) \rightarrow V_{h}$ associated with the graded meshes $\mathcal{T}_{h}$. For each node $\mathrm{n} \in \mathcal{N}_{h}$ we choose an edge $\ell_{\mathrm{n}} \in \mathcal{E}_{h}$ following the next rules:

Q.1 $\mathrm{n}$ is an endpoint of $\ell_{\mathrm{n}}$.

Q.2 $\ell_{\mathrm{n}} \subset \bar{\Omega}$ for every node $\mathrm{n}$.

Q.3 If $\mathrm{n} \in \bar{\Omega} \backslash \Gamma_{2}$ then $\ell_{\mathrm{n}}$ is a non-vertical edge.

Q.4 If $\mathrm{n}_{1}$ and $\mathrm{n}_{2}$ are nodes along the same vertical line, then the projections of $\ell_{\mathrm{n}_{1}}$ and $\ell_{\mathrm{n}_{2}}$ on the $x$-axis coincide.

Q.5 If $\mathrm{n} \in \Gamma_{2}$ then $\ell_{\mathrm{n}} \subset \Gamma_{2}$. 
For each edge $\ell \in \mathcal{E}_{h}$ let $\Pi_{\ell}: L^{2}(\ell) \rightarrow P_{1}(\ell)$ be the $L^{2}(\ell)$ projection on the space of linear polynomials on $\ell$. If $\mathrm{n} \in \mathcal{N}_{h}, \phi_{\mathrm{n}}$ denotes the Lagrangian basis associated with $\mathrm{n}$, that is, $\phi_{\mathrm{n}}$ is a piecewise linear function on $\mathcal{T}_{h}$ with $\phi_{\mathrm{n}}\left(\mathrm{n}^{\prime}\right)=\delta_{\mathrm{n}, \mathrm{n}^{\prime}}$ for every $\mathrm{n}^{\prime} \in \mathcal{N}_{h}$.

We are ready to introduce the operator $Q_{h}$ which, for a function $v \in H^{1}(\Omega)$, is defined by

$$
Q_{h} v=\sum_{\mathrm{n} \in \mathcal{N}_{h}} \Pi_{\ell_{\mathrm{n}}} v(\mathrm{n}) \phi_{\mathrm{n}}
$$

Following [25, Section 2], for each node $\mathrm{n}$ there exists a unique linear polynomial $\psi_{\mathrm{n}} \in P_{1}\left(\ell_{\mathrm{n}}\right)$, such that

$$
p(\mathrm{n})=\int_{\ell_{\mathrm{n}}} p \psi_{\mathrm{n}}, \quad \forall p \in P_{1}\left(\ell_{\mathrm{n}}\right) .
$$

Indeed, let $\hat{\ell}=[0,1]$, and $\hat{\psi}$ be the linear function such that

$$
\int_{\hat{\ell}} \hat{\psi} \hat{p}=\hat{p}(0), \quad \forall \hat{p} \in P_{1}([0,1]) \text {. }
$$

Explicitly, $\hat{\psi}(t)=-6 t+4, t \in[0,1]$. Then, if $\ell_{\mathrm{n}}=\overline{\mathrm{nn}^{\prime}}$ it follows that

$$
\psi_{\mathrm{n}}(x)=\left|\overline{\mathrm{nn}^{\prime}}\right|^{-1} \hat{\psi}(t), \quad \text { if } x=(1-t) \mathrm{n}+t \mathrm{n}^{\prime}, t \in[0,1] .
$$

Moreover, it holds (see also [25, Lemma 3.1])

$$
\left\|\psi_{\mathrm{n}}\right\|_{L^{\infty}\left(\ell_{\mathrm{n}}\right)} \leq 4\left|\ell_{\mathrm{n}}\right|^{-1}
$$

Now, taking $p=\Pi_{\ell_{\mathrm{n}}} v$ and using the definition of $\Pi_{\ell_{\mathrm{n}}}$, we have

$$
\Pi_{\ell_{\mathrm{n}}} v(\mathrm{n})=\int_{\ell_{\mathrm{n}}}\left(\Pi_{\ell_{\mathrm{n}}} v\right) \psi_{\mathrm{n}}=\int_{\ell_{\mathrm{n}}} v \psi_{\mathrm{n}}
$$

from where we can write $([25$, equation $(2.13)])$

$$
Q_{h} v=\sum_{\mathrm{n} \in \mathcal{N}_{h}}\left(\int_{\ell_{\mathrm{n}}} v \psi_{\mathrm{n}}\right) \phi_{\mathrm{n}}
$$

Proposition 4 There exists a constant $C$, depending only on the maximum angle of $\mathcal{T}_{h}$, such that for all $v \in H^{1}(\Omega)$

$$
\left\|\nabla Q_{h} v\right\|_{L^{2}(\Omega)} \leq C\|\nabla v\|_{L^{2}(\Omega)}
$$

Proof. Let $E \in \mathcal{T}_{h}^{\Omega}$ be an element with vertices $\mathrm{n}_{1}, \mathrm{n}_{2}$ and $\mathrm{n}_{3}$, and suppose that $\overline{\mathrm{n}_{2} \mathrm{n}_{3}}$ is the vertical edge of $E$. Then, for a constant $C$, which depends only on the maximum angle of $E$, we have

$$
\left\|\nabla Q_{h} v\right\|_{L^{2}(E)} \leq C\left(\left\|\partial_{y} Q_{h} v\right\|_{L^{2}(E)}+\left\|\partial_{\overline{n_{1} n_{2}}} Q_{h} v\right\|_{L^{2}(E)}\right)
$$

where $\partial_{\overline{n_{1} \mathrm{n}_{2}}}(\cdot)=\nabla(\cdot) \cdot \frac{\overline{\mathrm{n}_{1} \mathrm{n}_{2}}}{\left|\overline{\mathrm{n}_{1} \mathrm{n}_{2}}\right|}$. Let start with the estimate for $\left\|\partial_{y} Q_{h} v\right\|_{L^{2}(E)}$. We first assume that $E$ has no edge on $\Gamma_{2}$. We have

$$
\partial_{y} Q_{h} v=\left|\overline{\mathrm{n}_{2} \mathrm{n}_{3}}\right|^{-1}\left(Q_{h} v\left(\mathrm{n}_{3}\right)-Q_{h} v\left(\mathrm{n}_{2}\right)\right)=\left|\overline{\mathrm{n}_{2} \mathrm{n}_{3}}\right|^{-1}\left(\int_{\ell_{\mathrm{n}_{3}}} v \psi_{\mathrm{n}_{3}}-\int_{\ell_{\mathrm{n}_{2}}} v \psi_{\mathrm{n}_{2}}\right) .
$$

Suppose that $\ell_{\mathrm{n}_{2}}=\overline{\mathrm{n}_{2} \mathrm{n}_{2}^{\prime}}$ and $\ell_{\mathrm{n}_{3}}=\overline{\mathrm{n}_{3} \mathrm{n}_{3}^{\prime}}$. Then

$$
\int_{\ell_{\mathrm{n}_{2}}} v \psi_{\mathrm{n}_{2}}=\int_{0}^{1} v\left((1-t) \mathrm{n}_{2}+t \mathrm{n}_{2}^{\prime}\right) \psi_{\mathrm{n}_{2}}\left((1-t) \mathrm{n}_{2}+t \mathrm{n}_{2}^{\prime}\right)\left|\overline{\mathrm{n}_{2} \mathrm{n}_{2}^{\prime}}\right| d t
$$


and

$$
\int_{\ell_{\mathrm{n}_{3}}} v \psi_{\mathrm{n}_{3}}=\int_{0}^{1} v\left((1-t) \mathrm{n}_{3}+t \mathrm{n}_{3}^{\prime}\right) \psi_{\mathrm{n}_{3}}\left((1-t) \mathrm{n}_{3}+t \mathrm{n}_{3}^{\prime}\right)\left|\overline{\mathrm{n}_{3} \mathrm{n}_{3}^{\prime}}\right| d t .
$$

Taking into account assumptions Q.4 and Q.3, and property (12), we have

$$
\psi_{\mathrm{n}_{2}}\left((1-t) \mathrm{n}_{2}+t \mathrm{n}_{2}^{\prime}\right)\left|\overline{\mathrm{n}_{2} \mathrm{n}_{2}^{\prime}}\right|=\psi_{\mathrm{n}_{3}}\left((1-t) \mathrm{n}_{3}+t \mathrm{n}_{3}^{\prime}\right)\left|\overline{\mathrm{n}_{3} \mathrm{n}_{3}^{\prime}}\right|, \quad \forall t \in[0,1]
$$

and then we can write

$$
\partial_{y} Q_{h} v=\left|\overline{\mathrm{n}_{2} \mathrm{n}_{3}}\right|^{-1} \int_{0}^{1}\left[v\left((1-t) \mathrm{n}_{3}+t \mathrm{n}_{3}^{\prime}\right)-v\left((1-t) \mathrm{n}_{2}+t \mathrm{n}_{2}^{\prime}\right)\right] \psi_{\mathrm{n}_{3}}\left((1-t) \mathrm{n}_{3}+t \mathrm{n}_{3}^{\prime}\right)\left|\overline{\mathrm{n}_{3} \mathrm{n}_{3}^{\prime}}\right| d t .
$$

Using (13) we obtain

$$
\left|\partial_{y} Q_{h} v\right| \leq 4\left|\overline{\mathrm{n}_{2} \mathrm{n}_{3}}\right|^{-1} \int_{0}^{1}\left|v\left((1-t) \mathrm{n}_{3}+t \mathrm{n}_{3}^{\prime}\right)-v(1-t) \mathrm{n}_{2}+t \mathbf{n}_{2}^{\prime}\right| d t .
$$

From Q.4 it also follows that the first component of the points $(1-t) \mathrm{n}_{2}+t \mathrm{n}_{2}^{\prime}$ and $(1-t) \mathrm{n}_{3}+t \mathrm{n}_{3}^{\prime}$ coincide for all $t \in[0,1]$ and therefore, if $D_{E}^{2}$ denotes the subset of $\Omega$ limited by $\overline{\mathrm{n}_{2} \mathrm{n}_{3}}, \ell_{\mathrm{n}_{2}}, \ell_{\mathrm{n}_{3}}$ and a vertical line (or eventually just a point), we have

$$
\left|\partial_{y} Q_{h} v\right| \leq 4\left|\overline{\mathrm{n}_{2} \mathrm{n}_{3}}\right|^{-1}\left|\left(\mathrm{n}_{3}^{\prime}-\mathrm{n}_{3}\right)_{1}\right|^{-1} \iint_{D_{E}^{2}}\left|\partial_{y} v\right| .
$$

We remark that, $\left(\mathrm{n}_{3}^{\prime}-\mathrm{n}_{3}\right)_{1}$ denotes the first component of $\mathrm{n}_{3}^{\prime}-\mathrm{n}_{3}$. Hence, using Cauchy-Schwarz inequality we have

$$
\begin{aligned}
\left\|\partial_{y} Q_{h} v\right\|_{L^{2}(E)} & \leq 4|E|^{\frac{1}{2}}\left|\overline{\mathrm{n}_{2} \mathrm{n}_{3}}\right|^{-1}\left|\left(\mathrm{n}_{3}^{\prime}-\mathrm{n}_{3}\right)_{1}\right|^{-1} \iint_{D_{E}^{2}}\left|\partial_{y} v\right| \\
& \leq C|E|\left|\overline{\mathrm{n}_{2} \mathrm{n}_{3}}\right|^{-1}\left|\left(\mathrm{n}_{3}^{\prime}-\mathrm{n}_{3}\right)_{1}\right|^{-1}\left\|\partial_{y} v\right\|_{L^{2}\left(D_{E}^{2}\right)} \\
& \leq C\left\|\partial_{y} v\right\|_{L^{2}\left(\omega_{E}\right)}
\end{aligned}
$$

where we used (6).

Now we deal with $\partial_{\overline{\mathrm{n}_{1} \mathrm{n}_{2}}} Q_{h} v$. We have

$$
\partial_{\overline{\mathrm{n}_{1} \mathrm{n}_{2}}} Q_{h} v=\left|\overline{\mathrm{n}_{1} \mathrm{n}_{2}}\right|^{-1}\left[Q_{h} v\left(\mathrm{n}_{2}\right)-Q_{h} v\left(\mathrm{n}_{1}\right)\right]=\left|\overline{\mathrm{n}_{1} \mathrm{n}_{2}}\right|^{-1}\left[\Pi_{\ell_{\mathrm{n}_{2}}} v\left(\mathrm{n}_{2}\right)-\Pi_{\ell_{\mathrm{n}_{1}}} v\left(\mathrm{n}_{1}\right)\right] .
$$

Since for all edge $\ell, \Pi_{\ell} p=p$ for $P_{1}(\ell)$-functions $p$, and in particular, for constant functions, we can write

$$
\partial_{\overline{\mathrm{n}_{1} \mathrm{n}_{2}}} Q_{h} v=\left|\overline{\mathrm{n}_{1} \mathrm{n}_{2}}\right|^{-1}\left[\Pi_{\sigma_{\mathrm{n}_{2}}}\left(v-c_{E}\right)\left(\mathrm{n}_{2}\right)-\Pi_{\ell_{\mathrm{n}_{1}}}\left(v-c_{E}\right)\left(\mathrm{n}_{1}\right)\right]
$$

where $c_{E}$ is a constant to be chosen later. We consider now the case when $\ell_{\mathrm{n}_{2}} \not \subset \Gamma_{2}$. We note that $\mathrm{n}_{2} \neq(0,0)$ and so, from Q.4 and Q.3, $\omega_{\ell_{\mathrm{n}_{2}}}$ always contains a parallelogram with sides $\ell_{\mathrm{n}_{2}}$ and a vertical one of length $c\left|\overline{\mathrm{n}_{2} \mathrm{n}_{3}}\right|$ with $c$ independent of $E$ and $h$. Furthermore we can also assume that that parallelogram is contained in $\omega_{E}^{\prime}$. Then we can use a trace inequality to obtain

$$
\left\|v-c_{E}\right\|_{L^{2}\left(\ell_{2}\right)} \leq C\left(\left|\overline{\mathrm{n}_{2} \mathrm{n}_{3}}\right|^{-\frac{1}{2}}\left\|v-c_{E}\right\|_{L^{2}\left(\omega_{E}^{\prime}\right)}+\left|\overline{\mathrm{n}_{2} \mathrm{n}_{3}}\right|^{\frac{1}{2}}\left\|\partial_{y} v\right\|_{L^{2}\left(\omega_{E}^{\prime}\right)}\right) .
$$

It follows from (6) that $\left|\ell_{\mathrm{n}_{1}}\right|,\left|\ell_{\mathrm{n}_{2}}\right| \sim\left|\overline{\mathrm{n}_{1} \mathrm{n}_{2}}\right|$. Then, by (13), we have

$$
\begin{aligned}
\left|\Pi_{\ell_{\mathrm{n}_{2}}}\left(v-c_{E}\right)\left(\mathrm{n}_{2}\right)\right| & =\left|\int_{\ell_{\mathrm{n}_{2}}}\left(v-c_{E}\right) \psi_{\ell_{\mathrm{n}_{2}}}\right| \leq C\left|\overline{\mathrm{n}_{1} \mathrm{n}_{2}}\right|^{-1} \int_{\ell_{\mathrm{n}_{2}}}\left|v-c_{E}\right| \\
& \leq C\left|\overline{\mathrm{n}_{1} \mathrm{n}_{2}}\right|^{-1}\left|\ell_{\mathrm{n}_{2}}\right|^{\frac{1}{2}}\left\|v-c_{E}\right\|_{L^{2}\left(\ell_{\mathrm{n}_{2}}\right)} \\
& \leq C\left|\overline{\mathrm{n}_{1} \mathrm{n}_{2}}\right|^{-\frac{1}{2}}\left(\left|\overline{\mathrm{n}_{2} \mathrm{n}_{3}}\right|^{-\frac{1}{2}}\left\|v-c_{E}\right\|_{L^{2}\left(\omega_{E}^{\prime}\right)}+\left|\overline{\mathrm{n}_{2} \mathrm{n}_{3}}\right|^{\frac{1}{2}}\left\|\partial_{y} v\right\|_{L^{2}\left(\omega_{E}^{\prime}\right)}\right) .
\end{aligned}
$$


If $\mathrm{n}_{1} \neq(0,0)$ a similar estimate can be obtained for $\left|\Pi_{\ell_{\mathrm{n}_{1}}}\left(v-c_{E}\right)\left(\mathrm{n}_{1}\right)\right|$ and therefore

$$
\left|\partial_{\overline{\mathrm{n}_{1} \mathrm{n}_{2}}} Q_{h} v\right| \leq C\left|\overline{\mathrm{n}_{1} \mathrm{n}_{2}}\right|^{-\frac{3}{2}}\left(\left|\overline{\mathrm{n}_{2} \mathrm{n}_{3}}\right|^{-\frac{1}{2}}\left\|v-c_{E}\right\|_{L^{2}\left(\omega_{E}^{\prime}\right)}+\left|\overline{\mathrm{n}_{2} \mathrm{n}_{3}}\right|^{\frac{1}{2}}\left\|\partial_{y} v\right\|_{L^{2}\left(\omega_{E}^{\prime}\right)}\right),
$$

and so

$$
\left\|\partial_{\overline{\mathrm{n}_{1} \mathrm{n}_{2}}} Q_{h} v\right\|_{L^{2}(E)} \leq C|E|^{\frac{1}{2}}\left|\overline{\mathrm{n}_{1} \mathrm{n}_{2}}\right|^{-\frac{3}{2}}\left(\left|\overline{\mathrm{n}_{2} \mathrm{n}_{3}}\right|^{-\frac{1}{2}}\left\|v-c_{E}\right\|_{L^{2}\left(\omega_{E}^{\prime}\right)}+\left|\overline{\mathrm{n}_{2} \mathrm{n}_{3}}\right|^{\frac{1}{2}}\left\|\partial_{y} v\right\|_{L^{2}\left(\omega_{E}^{\prime}\right)}\right) .
$$

Now we can take $c_{E}$ following Proposition 3 to have

$$
\left\|v-c_{E}\right\|_{L^{2}\left(\omega_{E}^{\prime}\right)} \leq C\left(\left|\overline{\mathrm{n}_{1} \mathrm{n}_{2}}\right|\left\|\partial_{x} v\right\|_{L^{2}\left(\omega_{E}^{\prime}\right)}+\left|\overline{\mathrm{n}_{2} \mathrm{n}_{3}}\right|\left\|\partial_{y} v\right\|_{L^{2}\left(\omega_{E}^{\prime}\right)}\right) .
$$

Inserting this inequality in (16) and taking into account that $|E| \sim\left|\overline{\mathrm{n}_{1} \mathrm{n}_{2}}\right|\left|\overline{\mathrm{n}_{2} \mathrm{n}_{3}}\right|$ we obtain

$$
\begin{aligned}
\left\|\partial_{\overline{\mathrm{n}_{1} \mathrm{n}_{2}}} Q_{h} v\right\|_{L^{2}(E)} & \leq C\left(\left\|\partial_{x} v\right\|_{L^{2}\left(\omega_{E}^{\prime}\right)}+\frac{\left|\overline{\mathrm{n}_{2} \mathrm{n}_{3}}\right|}{\left|\overline{\bar{n}_{1} \mathrm{n}_{2}}\right|}\left\|\partial_{y} v\right\|_{L^{2}\left(\omega_{E}^{\prime}\right)}\right) \\
& \leq C\left(\left\|\partial_{x} v\right\|_{L^{2}\left(\omega_{E}\right)}+\left\|\partial_{y} v\right\|_{L^{2}\left(\omega_{E}\right)}\right),
\end{aligned}
$$

where we used that $\left|\overline{\mathrm{n}_{2} \mathrm{n}_{3}}\right| \leq C\left|\overline{\mathrm{n}_{1} \mathrm{n}_{2}}\right|$ thanks to assumption M.6.

Now we consider the case in which $E$ has the edge $\overline{\mathrm{n}_{2} \mathrm{n}_{3}}$ on $\Gamma_{2}$. In this case, $\ell_{\mathrm{n}_{2}}$ and $\ell_{\mathrm{n}_{3}}$ are both contained in $\Gamma_{2}$ and, on the other hand we know from condition M.6 since $\bar{E} \cap \Gamma_{2} \neq \emptyset$, that $\left|\overline{n_{2} n_{3}}\right| \sim\left|\ell_{n_{2}}\right|$, $\left|\overline{\mathrm{n}_{2} \mathrm{n}_{3}}\right| \sim\left|\ell_{\mathrm{n}_{3}}\right|,\left|\overline{\mathrm{n}_{2} \mathrm{n}_{3}}\right| \sim\left|\overline{\mathrm{n}_{1} \mathrm{n}_{2}}\right|$ and $\overline{\mathrm{n}_{2} \mathrm{n}_{3}} \sim h_{E}$. We have, again for a constant $c_{E}$ to be chosen later,

$$
\partial_{y} Q_{h} v=\left|\overline{\mathrm{n}_{2} \mathrm{n}_{3}}\right|^{-1}\left(\int_{\ell_{\mathrm{n}_{3}}}\left(v-c_{E}\right) \psi_{\mathrm{n}_{3}}-\int_{\ell_{\mathrm{n}_{2}}}\left(v-c_{E}\right) \psi_{\mathrm{n}_{2}}\right),
$$

and using the estimates for $\left|\psi_{\mathbf{n}_{2}}\right|$ and $\left|\psi_{\mathbf{n}_{3}}\right|$ and trace inequalities we obtain

$$
\begin{aligned}
\left|\partial_{y} Q_{h} v\right| & \leq C h_{E}^{-2}\left(\int_{\sigma_{\mathrm{n}_{2}}}\left|v-c_{E}\right|+\int_{\sigma_{\mathrm{n}_{3}}}\left|v-c_{E}\right|\right) \\
& \leq C\left(h_{E}^{-2}\left\|v-c_{E}\right\|_{L^{2}\left(\omega_{E}\right)}+h_{E}^{-1}\|\nabla v\|_{L^{2}\left(\omega_{E}\right)}\right),
\end{aligned}
$$

and now, taking $c_{E}$ as the mean value of $v$ on an element contained in $\omega_{E}$, by applying Poincaré inequality we have

from where we finally obtain

$$
\left|\partial_{y} Q_{h} v\right| \leq C h_{E}^{-1}\|\nabla v\|_{L^{2}\left(\omega_{E}\right)},
$$

$$
\left\|\partial_{y} Q_{h} v\right\|_{L^{2}(E)} \leq C\|\nabla v\|_{L^{2}\left(\omega_{E}\right)} .
$$

Clearly for this case, with $\overline{\mathrm{n}_{2} \mathrm{n}_{3}} \subset \Gamma_{2}$, exploiting the shape regularity of $E$ (which follows from condition M.6) we similarly obtain

$$
\left\|\partial_{x} Q_{h} v\right\|_{L^{2}(E)} \leq C\|\nabla v\|_{L^{2}\left(\omega_{E}\right)},
$$

from where we have

$$
\left\|\nabla Q_{h} v\right\|_{L^{2}(E)} \leq C\|\nabla v\|_{L^{2}\left(\omega_{E}\right)} .
$$

It just remains to consider the case in which $E$ has $(0,0)$ as a vertex. We have yet dealt with $\left\|\partial_{y} Q_{h} v\right\|_{L^{2}(E)}$ obtaining inequality (15). So, it remains to estimate $\left\|\partial_{x} Q_{h} v\right\|_{L^{2}(E)}$. We put $\mathrm{n}_{1}=(0,0)$ and $\mathrm{n}_{2}$ and $\mathrm{n}_{3}$ the other two vertices, with $\left(\mathrm{n}_{2}\right)_{2}=0$. Again we have (now $\partial_{\overline{\mathrm{n}_{1} \mathrm{n}_{2}}}=\partial_{x}$ )

$$
\partial_{x} Q_{h} v=\left|\overline{\mathrm{n}_{1} \mathrm{n}_{2}}\right|^{-1}\left[\Pi_{\ell_{\mathrm{n}_{2}}}\left(v-c_{E}\right)\left(\mathrm{n}_{2}\right)-\Pi_{\ell_{\mathrm{n}_{1}}}\left(v-c_{E}\right)\left(\mathrm{n}_{1}\right)\right],
$$

for a constant $c_{E}$. We choose $c_{E}$ as the mean value of $v$ on a triangular element contained in $\omega_{E}^{\prime}$ as in Proposition 3. The first term inside the square brackets can be treated as before, using Poincaré and trace inequalities,

$$
\left|\Pi_{\ell_{\mathrm{n}_{2}}}\left(v-c_{E}\right)\left(\mathrm{n}_{2}\right)\right| \leq C\left[\left(\frac{\left|\overline{\mathrm{n}_{1} \mathrm{n}_{2}}\right|}{\left|\overline{\mathrm{n}_{2} \mathrm{n}_{3}}\right|}\right)^{\frac{1}{2}}\left\|\partial_{x} v\right\|_{L^{2}\left(\omega_{E}\right)}+\left(\frac{\left|\overline{\mathrm{n}_{2} \mathrm{n}_{3}}\right|}{\left|\overline{\mathrm{n}_{1} \mathrm{n}_{2}}\right|}\right)^{\frac{1}{2}}\left\|\partial_{y} v\right\|_{L^{2}\left(\omega_{E}\right)}\right] .
$$


On the other hand, by using the trace inequality [3, Ineq. (2.4)] we have

$$
\begin{aligned}
\left|\Pi_{\ell_{\mathrm{n}_{1}}}\left(v-c_{E}\right)\left(\mathrm{n}_{1}\right)\right| & =\left|\int_{\ell_{\mathrm{n}_{1}}}\left(v-c_{E}\right) \psi_{\mathrm{n}_{1}}\right| \\
& \leq C\left|\overline{\mathrm{n}_{1} \mathrm{n}_{2}}\right|^{-1}\left\|v-c_{E}\right\|_{L^{1}\left(\ell_{\mathrm{n}_{1}}\right)} \\
& \leq C\left|\overline{\mathrm{n}_{1} \mathrm{n}_{2}}\right|^{-1}\left(\left\|\left(v-c_{E}\right) x^{-\alpha}\right\|_{L^{1}(E)}+\|\nabla v\|_{L^{1}(E)}\right) \\
& \leq C\left|\overline{\mathrm{n}_{1} \mathrm{n}_{2}}\right|^{-1}\left(\left|\overline{\mathrm{n}_{1} \mathrm{n}_{2}}\right|^{-\frac{\alpha+1}{p}+1}\left\|v-c_{E}\right\|_{L^{p}(E)}+\left|\overline{\mathrm{n}_{1} \mathrm{n}_{2}}\right|^{\frac{\alpha+1}{2}}\|\nabla v\|_{L^{2}(E)}\right),
\end{aligned}
$$

where we used Hölder $p, q$ inequality with $p>1+\alpha,\left\|x^{-\alpha}\right\|_{L^{q}(E)}=\left|\overline{\mathrm{n}_{1} \mathrm{n}_{2}}\right|^{-\frac{\alpha+1}{p}+1}$ and $|E| \sim\left|\overline{\mathrm{n}_{1} \mathrm{n}_{2}}\right|^{\alpha+1}$. Now, in $E$ we have the scaled embedding inequality, which follows from the Sobolev embedding theorem in cusps [5, Theorem 5.35] by standard rescaling arguments

$$
\|w\|_{L^{p}(E)} \leq C\left|\overline{\mathrm{n}_{1} \mathrm{n}_{2}}\right|^{(\alpha+1) \frac{2-p}{2 p}}\left(\|w\|_{L^{2}(E)}+\left|\overline{\mathrm{n}_{1} \mathrm{n}_{2}}\right|\left\|\partial_{x} w\right\|_{L^{2}(E)}+\left|\overline{\mathrm{n}_{1} \mathrm{n}_{2}}\right|^{\alpha}\left\|\partial_{y} w\right\|_{L^{2}(E)}\right),
$$

valid for all $w \in H^{1}(E)$ and $p<6$. Then we obtain

$$
\begin{aligned}
\left|\Pi_{\ell_{\mathrm{n}_{1}}}\left(v-c_{E}\right)\left(\mathrm{n}_{1}\right)\right| \leq & \\
& \left|\overline{\mathrm{n}_{1} \mathrm{n}_{2}}\right|^{-\frac{\alpha+1}{2}}\left(\left|\overline{\mathrm{n}_{1} \mathrm{n}_{2}}\right|\left\|\partial_{x} v\right\|_{L^{2}(E)}+\left|\overline{\mathrm{n}_{1} \mathrm{n}_{2}}\right|^{\alpha}\left\|\partial_{y} v\right\|_{L^{2}(E)}\right)+\left|\overline{\mathrm{n}_{1} \mathrm{n}_{2}}\right|^{\frac{\alpha-1}{2}}\|\nabla v\|_{L^{2}(E)},
\end{aligned}
$$

where we have used the Poincaré inequality as stated in Remark 1. From (17)-(19), and since $|E| \sim$ $\left|\overline{\mathrm{n}_{1} \mathrm{n}_{2}}\right|^{\alpha+1}$ and $\left|\overline{\mathrm{n}_{2} \mathrm{n}_{3}}\right|=\left|\overline{\mathrm{n}_{1} \mathrm{n}_{2}}\right|^{\alpha}$ we have

$$
\begin{aligned}
&\left\|\partial_{x} Q_{h} v\right\|_{L^{2}(E)} \leq C\left|\overline{\mathrm{n}_{1} \mathrm{n}_{2}}\right|^{\frac{\alpha+1}{2}-1}\left(\left|\overline{\mathrm{n}_{1} \mathrm{n}_{2}}\right|^{\frac{1-\alpha}{2}}\left\|\partial_{x} v\right\|_{L^{2}\left(\omega_{E}\right)}+\left|\overline{\mathrm{n}_{1} \mathrm{n}_{2}}\right|^{\frac{\alpha-1}{2}}\left\|\partial_{y} v\right\|_{L^{2}\left(\omega_{E}\right)}\right) \\
&+C\left(\left\|\partial_{x} v\right\|_{L^{2}(E)}+\left|\overline{\mathrm{n}_{1} \mathrm{n}_{2}}\right|^{\alpha-1}\|\nabla v\|_{L^{2}(E)}\right) \\
& \leq C\left(\left\|\partial_{x} v\right\|_{L^{2}\left(\omega_{E}\right)}+\left|\overline{\mathrm{n}_{1} \mathrm{n}_{2}}\right|^{\alpha-1}\left\|\partial_{y} v\right\|_{L^{2}\left(\omega_{E}\right)}\right) .
\end{aligned}
$$

The proof conclude by adding the inequalities obtained for each element $E \in \mathcal{T}_{h}^{\Omega}$ and taking into account that

for all $E \in \mathcal{T}_{h}^{\Omega}$.

$$
\#\left\{E^{\prime} \in \mathcal{T}_{h}^{\Omega}: E \cap E^{\prime} \neq \emptyset\right\} \leq C,
$$

Corollary 2 There exists a constant $C$, depending only on the maximum angle of $\mathcal{T}_{h}$, such that for all $v \in H^{1}(\Omega)$ we have

$$
\left\|\nabla Q_{h} v\right\|_{L^{2}\left(\Omega_{h}\right)} \leq C\|\nabla v\|_{L^{2}(\Omega)} .
$$

Proof. We note that, for a constant $C$ which depends only on $\alpha$, if $K \in \mathcal{T}_{h}$ and $E=K \cap \Omega$ then

$$
|K| \leq C|E|
$$

Hence, taking into account that $\left.\nabla Q_{h} v\right|_{K} \in P_{0}(K)^{2}$, we have

$$
\left\|\nabla Q_{h} v\right\|_{L^{2}(K)}=\left.|K|^{\frac{1}{2}}\left|\nabla Q_{h} v\right|_{K}|\leq C| E\right|^{\frac{1}{2}}\left|\nabla Q_{h} v\right|_{K} \mid=C\left\|\nabla Q_{h} v\right\|_{L^{2}(E)} .
$$

Therefore

$$
\left\|\nabla Q_{h} v\right\|_{L^{2}\left(\Omega_{h} \backslash \Omega\right)} \leq C\left\|\nabla Q_{h} v\right\|_{L^{2}(\Omega)},
$$

which together with (14) gives (20).

Proposition 5 There exists a constant $C$, independent of $h$, such that the following interpolation error estimates hold:

$$
\begin{aligned}
& \left\|\nabla\left(v-Q_{h} v\right)\right\|_{L^{2}(\Omega)} \leq C|v|_{H^{1}(\Omega)}, \quad \forall v \in H^{1}(\Omega) \\
& \left\|\nabla\left(v-Q_{h} v\right)\right\|_{L^{2}(\Omega)} \leq C h\|v\|_{H^{2}(\Omega)}, \quad \forall v \in H^{2}(\Omega) .
\end{aligned}
$$


Proof. Using the triangle inequality and (14) we have, for all $v \in H^{1}(\Omega)$,

$$
\left\|\nabla\left(v-Q_{h} v\right)\right\|_{L^{2}(\Omega)} \leq\|\nabla v\|_{L^{2}(\Omega)}+\left\|\nabla Q_{h} v\right\|_{L^{2}(\Omega)} \leq C\|\nabla v\|_{L^{2}(\Omega)},
$$

which gives (21).

Denote by $\mathcal{L}_{h}: H^{2}(\Omega) \rightarrow V_{h}$ the Lagrange interpolation operator defined on the graded mesh $\mathcal{T}_{h}$. It follows from [1, Theorem 2.2 and equation (1.5)] that

$$
\left\|\nabla\left(v-\mathcal{L}_{h} v\right)\right\|_{L^{2}(\Omega)} \leq C h\|v\|_{H^{2}(\Omega)}, \quad \forall v \in H^{2}(\Omega) .
$$

Taking into account that for any function $w$ in $V_{h}$ and $\mathrm{n} \in \mathcal{N}_{h}, \Pi_{\ell} w(\mathrm{n})=w(\mathrm{n})$, we have that $Q_{h} \mathcal{L}_{h} v=\mathcal{L}_{h} v$. Then, using (14) and (23) we obtain

$$
\begin{aligned}
\left\|\nabla\left(v-Q_{h} v\right)\right\|_{L^{2}(\Omega)} & \leq\left\|\nabla\left(v-\mathcal{L}_{h} v\right)\right\|_{L^{2}(\Omega)}+\left\|\nabla\left(\mathcal{L}_{h} v-Q_{h} v\right)\right\|_{L^{2}(\Omega)} \\
& =\left\|\nabla\left(v-\mathcal{L}_{h} v\right)\right\|_{L^{2}(\Omega)}+\left\|\nabla Q_{h}\left(v-\mathcal{L}_{h} v\right)\right\|_{L^{2}(\Omega)} \\
& \leq\left\|\nabla\left(v-\mathcal{L}_{h} v\right)\right\|_{L^{2}(\Omega)}+C\left\|\nabla\left(v-\mathcal{L}_{h} v\right)\right\|_{L^{2}(\Omega)} \\
& \leq C h\|v\|_{H^{2}(\Omega)},
\end{aligned}
$$

which proves (22).

From the operator interpolation theory we obtain the next result.

Corollary 3 Let $s \in[0,1]$. There exists a constant $C$, independent of $h$, such that

$$
\left\|\nabla\left(v-Q_{h} v\right)\right\|_{L^{2}(\Omega)} \leq C h^{s}\|v\|_{H^{1+s}(\Omega)}, \quad \forall v \in H^{1+s}(\Omega) .
$$

Let $v \in H^{2}(\Omega)$. We know from [21, Section 5.4.2], with $l=p=n=2$ and $\varphi(x)=x^{\alpha}$, that there exists an extension $\tilde{v} \in H_{\alpha}^{2}\left(\mathbb{R}^{2}\right)$ such that

$$
\|\tilde{v}\|_{H_{\alpha}^{2}\left(\mathbb{R}^{2}\right)} \leq C\|v\|_{H^{2}(\Omega)} .
$$

where the weighted Sobolev space $H_{\alpha}^{2}(\mathcal{D})$ on a domain $\mathcal{D} \subset \mathbb{R}^{2}$ is defined as

$$
H_{\alpha}^{2}(\mathcal{D})=\left\{v: r^{\frac{\alpha-1}{2}} D^{\delta} v \in L^{2}(\mathcal{D}) \quad \forall \delta,|\delta| \leq 2\right\},
$$

with $r=\sqrt{x^{2}+y^{2}}$, and

$$
\|v\|_{H_{\alpha}^{2}(\mathcal{D})}^{2}=\sum_{|\delta| \leq 2}\left\|r^{\frac{\alpha-1}{2}} D^{\delta} v\right\|_{L^{2}(\mathcal{D})}^{2}
$$

Now, we show the following result for functions in $H_{\alpha}^{2}\left(\Omega_{h}\right)$ which will be useful later on.

Proposition 6 There exists a constant $C$, depending only on $\alpha$, such that

$$
\left\|\nabla\left(v-Q_{h} v\right)\right\|_{L^{2}\left(\Omega_{h}\right)} \leq C h\|v\|_{H_{\alpha}^{2}\left(\Omega_{h}\right)}, \quad \forall v \in H_{\alpha}^{2}\left(\Omega_{h}\right) .
$$

Proof. Using Corollary 2 we have

$$
\begin{aligned}
\left\|\nabla\left(v-Q_{h} v\right)\right\|_{L^{2}\left(\Omega_{h}\right)} & \leq\left\|\nabla\left(v-\mathcal{L}_{h} v\right)\right\|_{L^{2}\left(\Omega_{h}\right)}+\left\|\nabla\left(Q_{h}\left(v-\mathcal{L}_{h} v\right)\right)\right\|_{L^{2}\left(\Omega_{h}\right)} \\
& \leq\left\|\nabla\left(v-\mathcal{L}_{h} v\right)\right\|_{L^{2}\left(\Omega_{h}\right)}+C\left\|\nabla\left(v-\mathcal{L}_{h} v\right)\right\|_{L^{2}(\Omega)} \\
& \leq C\left\|\nabla\left(v-\mathcal{L}_{h} v\right)\right\|_{L^{2}\left(\Omega_{h}\right)}
\end{aligned}
$$

Now we use Theorem 2.2 of [1] and conclude the proof. 


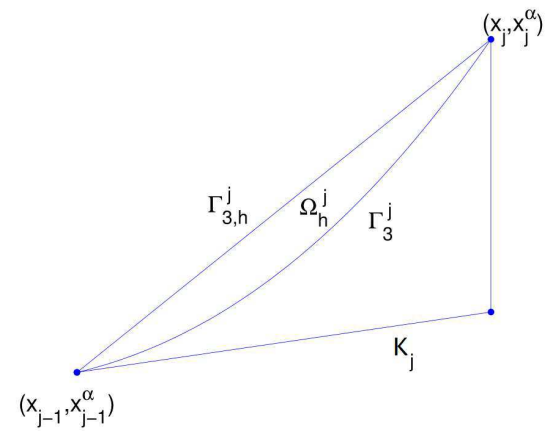

Fig. 3 The region $\Omega_{h}^{j}$ and the corresponding triangle $K_{j}$ satisfying (Ha).

\section{Finite element approximations}

For each $j=1, \ldots, n$ we denote by $\Gamma_{3, h}^{j}$ the edge on the boundary of $\Omega_{h}$ with endpoints $P_{j-1}=$ $\left(x_{j-1}, x_{j-1}^{\alpha}\right)$ and $P_{j}=\left(x_{j}, x_{j}^{\alpha}\right)$, being $x_{0}=0$ and $x_{n}=1$, and by $\Gamma_{3}^{j}$ the part of $\Gamma_{3}$ with the same endpoints (see Figure 3). By $\Omega_{h}^{j}$ we denote the region bounded by $\Gamma_{3}^{j}$ and $\Gamma_{3, h}^{j}$.

In addition to the assumptions M.1-M.6 we also assume the following hypothesis on the meshes:

(Ha) For $1 \leq j \leq n$ the region $\Omega_{h}^{j}$ is strictly contained in only one triangle denoted by $K_{j}$. We denote the diameter of $K_{j}$ by $h_{j}$.

We will show in Section 6 how meshes verifying conditions M1-M6 and (Ha) can indeed be constructed. We know, for these graded meshes, that the following properties hold (see [1, Lemma 2.1 and Lemma $2.3])$ :

P.1 For $2 \leq j \leq n, x_{j-1} \leq x_{j} \leq C x_{j-1}$ with $C$ depending only on $\alpha$ and $\tau$.

P.2 $\left|\Omega_{h} \backslash \Omega\right| \leq C h^{2}$.

P.3 Let $K \in \mathcal{T}_{h}$ be such that $K \cap \Gamma_{3} \neq \emptyset$, and put $E=K \cap \Omega$. Then $|E| \leq|K| \leq C|E|$ with $C$ independent of $K$ and $h$.

Proposition 7 Let $s \in[0,1]$. There exists a constant $C$, independent of $s$ and $h$ but might depend on $\alpha$, such that we have

$$
\left\|\nabla Q_{h} \phi\right\|_{L^{2}\left(\Omega_{h} \backslash \Omega\right)} \leq C\left(h \sqrt{\log \frac{1}{h}}\right)^{s}\|\phi\|_{H^{1+s}(\Omega)}, \quad \forall \phi \in H^{1+s}(\Omega) .
$$

Proof. From Corollary 2 we have

$$
\left\|\nabla Q_{h} v\right\|_{L^{2}\left(\Omega_{h} \backslash \Omega\right)} \leq C\|v\|_{H^{1}(\Omega)}, \quad \forall v \in H^{1}(\Omega) .
$$

Now, let $v \in H^{2}(\Omega)$. We know from [21, Section 5.4.2] that there exists an extension $\tilde{v} \in H_{\alpha}^{2}\left(\mathbb{R}^{2}\right)$ such that

$$
\|\tilde{v}\|_{H_{\alpha}^{2}\left(\mathbb{R}^{2}\right)} \leq C\|v\|_{H^{2}(\Omega)} .
$$

From Proposition 6, and since $Q_{h} v=Q_{h} \tilde{v}$ we have

$$
\left\|\nabla\left(\tilde{v}-Q_{h} v\right)\right\|_{L^{2}\left(\Omega_{h}\right)} \leq C h\|\tilde{v}\|_{H_{\alpha}^{2}\left(\Omega_{h}\right)} \leq C h\|v\|_{H^{2}(\Omega)} .
$$


Then

$$
\begin{aligned}
\left\|\nabla Q_{h} v\right\|_{L^{2}\left(\Omega_{h} \backslash \Omega\right)} & \leq\left\|\nabla\left(\tilde{v}-Q_{h} v\right)\right\|_{L^{2}\left(\Omega_{h} \backslash \Omega\right)}+\|\nabla \tilde{v}\|_{L^{2}\left(\Omega_{h} \backslash \Omega\right)} \\
& \leq C h\|v\|_{H^{2}(\Omega)}+\|\nabla \tilde{v}\|_{L^{2}\left(\Omega_{h} \backslash \Omega\right)} .
\end{aligned}
$$

From [4, Lemma 4.1] we have

$$
\|\nabla \tilde{v}\|_{L^{2}\left(\Omega_{h} \backslash \Omega\right)} \leq C h \sqrt{\log \frac{1}{h}}\|v\|_{H^{2}(\Omega)},
$$

from where we obtain

$$
\left\|\nabla Q_{h} v\right\|_{L^{2}\left(\Omega_{h} \backslash \Omega\right)} \leq C h \sqrt{\log \frac{1}{h}}\|v\|_{H^{2}(\Omega)} .
$$

So we proved

$$
\left\|\nabla Q_{h} v\right\|_{L^{2}\left(\Omega_{h} \backslash \Omega\right)} \leq C h \sqrt{\log \frac{1}{h}}\|v\|_{H^{2}(\Omega)}, \quad \forall v \in H^{2}(\Omega) .
$$

Inequality (24) follows from (25) and (26) by the operator interpolation theory.

Now, we will obtain error estimates for the solutions of the source problems (3) and (5) in $L^{2}\left(\Gamma_{3}\right)-$ norm.

Theorem 3 Assume $1<\alpha<2$ and $\sigma=1-\frac{\alpha}{2}$. Let $u$ and $u_{h}$ be the solutions of problems (3) and (5) respectively. If $g \in H^{s}\left(\Gamma_{3}\right)$, with $0 \leq s<\frac{1}{2}$, then there exists a positive constant $C$, dependent on $\alpha$ but independent of $s$ and $h$, such that

$$
\left\|u-u_{h}\right\|_{L^{2}\left(\Gamma_{3}\right)} \leq C\left(h \sqrt{\log \left(\frac{1}{h}\right)}\right)^{\frac{\sigma}{2}+s}\|g\|_{H^{s}\left(\Gamma_{3}\right)} .
$$

Proof. Let $e=u-u_{h}$. In order to obtain error estimates in $L^{2}\left(\Gamma_{3}\right)$ we consider the following auxiliary problem:

$$
\begin{aligned}
-\triangle \phi & =0 & & \text { in } \Omega, \\
\frac{\partial \phi}{\partial \nu} & =0 & & \text { on } \Gamma_{1}, \\
\phi & =0 & & \text { on } \Gamma_{2}, \\
\frac{\partial \phi}{\partial \nu} & =e & & \text { on } \Gamma_{3} .
\end{aligned}
$$

Then,

$$
\begin{aligned}
\|e\|_{L^{2}\left(\Gamma_{3}\right)}^{2} & =\int_{\Gamma_{3}} e^{2}=\int_{\Gamma_{3}} \frac{\partial \phi}{\partial \nu} e=\int_{\Omega} \nabla \phi \nabla e \\
& =\int_{\Omega} \nabla\left(\phi-Q_{h} \phi\right) \nabla e+\int_{\Omega} \nabla\left(Q_{h} \phi\right) \nabla e
\end{aligned}
$$

Now, from the error equation we know that for any $v \in V_{h}$ we have

$$
\begin{aligned}
\int_{\Omega} \nabla e \cdot \nabla v & =\int_{\Gamma_{3}} g v-\int_{\Omega} \nabla u_{h} \cdot \nabla v \\
& =\int_{\Gamma_{3}} g v-\int_{\Omega_{h}} \nabla u_{h} \nabla v+\int_{\Omega_{h} \backslash \Omega} \nabla u_{h} \nabla v \\
& =\int_{\Omega_{h} \backslash \Omega} \nabla u_{h} \nabla v .
\end{aligned}
$$


Therefore,

$$
\|e\|_{L^{2}\left(\Gamma_{3}\right)}^{2} \leq\|\nabla e\|_{L^{2}(\Omega)}\left\|\nabla\left(\phi-Q_{h} \phi\right)\right\|_{L^{2}(\Omega)}+\left\|\nabla u_{h}\right\|_{L^{2}\left(\Omega_{h} \backslash \Omega\right)}\left\|\nabla Q_{h} \phi\right\|_{L^{2}\left(\Omega_{h} \backslash \Omega\right)}
$$

Since $e \in H^{1}(\Omega)$ from (4) we know that $\left.e\right|_{\Gamma_{3}} \in H^{\sigma}\left(\Gamma_{3}\right)$. In view of Theorem $2, \phi \in H^{1+\sigma}(\Omega)$, and in particular, $\phi \in H^{1+\frac{\sigma}{2}}(\Omega)$. Using the interpolation error estimates obtained in Corollary 3 we get

$$
\|e\|_{L^{2}\left(\Gamma_{3}\right)}^{2} \leq C h^{\frac{\sigma}{2}}\|\phi\|_{H^{1+\frac{\sigma}{2}(\Omega)}}\|\nabla e\|_{L^{2}(\Omega)}+\left\|\nabla u_{h}\right\|_{L^{2}\left(\Omega_{h} \backslash \Omega\right)}\left\|\nabla Q_{h} \phi\right\|_{L^{2}\left(\Omega_{h} \backslash \Omega\right)} .
$$

Using Proposition 7 we obtain

$$
\|e\|_{L^{2}\left(\Gamma_{3}\right)}^{2} \leq C h^{\frac{\sigma}{2}}\|\phi\|_{H^{1+\frac{\sigma}{2}}(\Omega)}\left[\|\nabla e\|_{L^{2}(\Omega)}+\left(\sqrt{\log \frac{1}{h}}\right)^{\frac{\sigma}{2}}\left\|\nabla u_{h}\right\|_{L^{2}\left(\Omega_{h} \backslash \Omega\right)}\right] .
$$

So

$$
\|e\|_{L^{2}\left(\Gamma_{3}\right)}^{2} \leq C\left(h \sqrt{\log \frac{1}{h}}\right)^{\frac{\sigma}{2}}\|\phi\|_{H^{1+\frac{\sigma}{2}(\Omega)}}\left(\|\nabla e\|_{L^{2}(\Omega)}+\left\|\nabla u_{h}\right\|_{L^{2}\left(\Omega_{h}\right)}\right),
$$

and for $1 \leq \alpha<2$ we have from the definitions of $u$ and $u_{h}$ and a trace inequality

$$
\|e\|_{L^{2}\left(\Gamma_{3}\right)}^{2} \leq C\left(h \sqrt{\log \frac{1}{h}}\right)^{\frac{\sigma}{2}}\|\phi\|_{H^{1+\frac{\sigma}{2}(\Omega)}}\|g\|_{L^{2}\left(\Gamma_{3}\right)} .
$$

Now, we can apply similar arguments to those given in the proof of Proposition 4.4 of [11]. Indeed, from Theorem 2 we know that $\phi \in H^{1+\sigma}(\Omega)$, with $\sigma=1-\frac{\alpha}{2}$ and $\|\phi\|_{H^{1+\sigma}(\Omega)} \leq C\left\|_{e}\right\|_{H^{\sigma}\left(\Gamma_{3}\right)}$. On the other hand, the problem (3) is well defined for any $e \in H^{-\sigma}\left(\stackrel{2}{\Gamma_{3}}\right)$ and $\|\phi\|_{H^{1}(\Omega)} \leq C\|e\|_{H^{-\sigma}\left(\Gamma_{3}\right)}$. Therefore, by interpolation of Sobolev spaces, we can conclude that

$$
\|\phi\|_{H^{1+\frac{\sigma}{2}}(\Omega)} \leq C\|e\|_{L^{2}\left(\Gamma_{3}\right)} .
$$

Then

$$
\|e\|_{L^{2}\left(\Gamma_{3}\right)}^{2} \leq C\left(h \sqrt{\log \frac{1}{h}}\right)^{\frac{\sigma}{2}}\|e\|_{L^{2}\left(\Gamma_{3}\right)}\|g\|_{L^{2}\left(\Gamma_{3}\right)},
$$

which proves the assertion for $s=0$.

It was proved in [4, Theorem 4.2] (and its proof) that if $g \in H^{1}\left(\Gamma_{3}\right)$ with $g(0,0)=0$ then

$$
\|\nabla e\|_{L^{2}(\Omega)} \leq\|\nabla e\|_{L^{2}\left(\Omega_{h}\right)} \leq C h \sqrt{\log \frac{1}{h}}\|g\|_{H^{1}\left(\Gamma_{3}\right)}
$$

and using this and also [4, Lemma 4.1] we have (always for $\alpha<2$ )

$$
\begin{aligned}
\left\|\nabla u_{h}\right\|_{L^{2}\left(\Omega_{h} \backslash \Omega\right)} & \leq\left\|\nabla\left(u_{h}-\tilde{u}\right)\right\|_{L^{2}\left(\Omega_{h} \backslash \Omega\right)}+\|\nabla \tilde{u}\|_{L^{2}\left(\Omega_{h} \backslash \Omega\right)} \\
& \leq C h \sqrt{\log \frac{1}{h}}\|g\|_{H^{1}\left(\Gamma_{3}\right)} .
\end{aligned}
$$

On the other hand we also have (again for $\alpha<2$ )

$$
\|\nabla e\|_{L^{2}(\Omega)} \leq C\|g\|_{L^{2}\left(\Gamma_{3}\right)}
$$

and

Recalling that

$$
\left.\left\|\nabla u_{h}\right\|_{L^{2}\left(\Omega_{h} \backslash \Omega\right)} \leq C\left\|\nabla u_{h}\right\|_{L^{2}(\Omega)} \leq C\|g\|_{L^{2}\left(\Gamma_{3}\right.}\right) .
$$

$$
0 \leq s<\frac{1}{2} \quad \Longrightarrow \quad H^{s}\left(\Gamma_{3}\right)=\left[L^{2}\left(\Gamma_{3}\right), \stackrel{o}{\left.H^{1}\left(\Gamma_{3}\right)\right]_{s}}\right.
$$


by interpolation we obtain

$$
\|\nabla e\|_{L^{2}(\Omega)} \leq C\left(h \sqrt{\log \frac{1}{h}}\right)^{s}\|g\|_{H^{s}\left(\Gamma_{3}\right)}
$$

and

$$
\left\|\nabla u_{h}\right\|_{L^{2}\left(\Omega_{h} \backslash \Omega\right)} \leq C\left(h \sqrt{\log \frac{1}{h}}\right)^{s}\|g\|_{H^{s}\left(\Gamma_{3}\right)} .
$$

Inserting these inequalities in (27) we arrive at

$$
\begin{aligned}
\|e\|_{L^{2}\left(\Gamma_{3}\right)}^{2} & \leq C\left(h \sqrt{\log \frac{1}{h}}\right)^{\frac{\sigma}{2}+s}\|\phi\|_{H^{1+\frac{\sigma}{2}}(\Omega)}\|g\|_{H^{s}\left(\Gamma_{3}\right)} \\
& \leq C\left(h \sqrt{\log \frac{1}{h}}\right)^{\frac{\sigma}{2}+s}\|e\|_{L^{2}\left(\Gamma_{3}\right)}\|g\|_{H^{s}\left(\Gamma_{3}\right)},
\end{aligned}
$$

which proves the assertion for $s>0$.

\section{Spectral approximation}

In this section we prove the convergence of the eigenfunctions and eigenvalues. In what follows we assume that $1<\alpha<2$ and $\sigma=1-\frac{\alpha}{2}$.

Next Lemma, which is a direct consequence of Theorem 3, shows that $T_{h}$ converge to $T$ in operator norm when $h$ goes to zero.

Lemma 1 There exists a positive constant $C$ such that for any $g \in L^{2}\left(\Gamma_{3}\right)$

$$
\left\|T g-T_{h} g\right\|_{L^{2}\left(\Gamma_{3}\right)} \leq C\left(h \sqrt{\log \frac{1}{h}}\right)^{\frac{\sigma}{2}}\|g\|_{L^{2}\left(\Gamma_{3}\right)} .
$$

Despite of the poor order given in (30) this result allows us to state the convergence for eigenvalues. In the following theorem we study the regularity of the eigenfunctions of problem (1). In Particular, it shows that, for any $\alpha \in(1,2)$ the eigenfunctions are always in $H^{\frac{1}{2}}\left(\Gamma_{3}\right)$.

Theorem 4 For all $\epsilon>0$, the restrictions to $\Gamma_{3}$ of eigenfunctions of problem (1) are in $H^{\frac{1}{2}+\sigma-\epsilon}\left(\Gamma_{3}\right)$.

Proof. Let $\gamma$ be the trace operator on $\Gamma_{3}$. From Theorem 1 we know that

$$
\gamma: H^{1}(\Omega) \rightarrow H^{\sigma}\left(\Gamma_{3}\right)
$$

and from which one can deduce that also

$$
\gamma: H^{2}(\Omega) \rightarrow H^{1+\sigma}\left(\Gamma_{3}\right)
$$

Therefore, by interpolation we have

$$
\gamma: H^{1+s}(\Omega) \rightarrow H^{\sigma+s}\left(\Gamma_{3}\right), \quad s \in[0,1] .
$$

On the other hand, denoting $\stackrel{o}{H^{1}}\left(\Gamma_{3}\right)=\left\{g \in H^{1}\left(\Gamma_{3}\right): g(0,0)=0\right\}$, we know from [4] that the operator $B$, introduced at the beginning of Section 2 , verifies

$$
B: \stackrel{o}{H^{1}}\left(\Gamma_{3}\right) \rightarrow H^{2}(\Omega)
$$


and from Theorem 2 that

$$
B: H^{\sigma}\left(\Gamma_{3}\right) \rightarrow H^{1+\sigma}(\Omega) .
$$

Then we have

$$
B: H^{s}\left(\Gamma_{3}\right) \rightarrow H^{1+s}(\Omega), \quad \sigma \leq s<\frac{1}{2} .
$$

Let $u \in H^{1}(\Omega)$ be an eigenfunction of (1). Then from Theorem 1 we know that $\left.u\right|_{\Gamma_{3}} \in H^{\sigma}\left(\Gamma_{3}\right)$. It follows from Theorem 2 that $u \in H^{1+\sigma}(\Omega)$. Then from (31) we have $\left.u\right|_{\Gamma_{3}} \in H^{2 \sigma}\left(\Gamma_{3}\right)$.

If $2 \sigma>\frac{1}{2}$ then we have

$$
\left.u\right|_{\Gamma_{3}} \in H^{\frac{1}{2}-\epsilon}\left(\Gamma_{3}\right)=\left[L^{2}\left(\Gamma_{3}\right), \stackrel{o}{H^{1}}\left(\Gamma_{3}\right)\right]_{\frac{1}{2}-\epsilon} .
$$

Now, from (32) we have $u \in H^{\frac{3}{2}-\epsilon}(\Omega)$. Using again (31) we obtain that $u \in H^{\frac{1}{2}+\sigma-\epsilon}\left(\Gamma_{3}\right)$.

If $2 \sigma<\frac{1}{2}$ we obtain from $(32)$ that $u \in H^{1+2 \sigma}(\Omega)$, and so, from $\left.(31) u\right|_{\Gamma_{3}} \in H^{3 \sigma}\left(\Gamma_{3}\right)$. Now we conclude as before if $3 \sigma>\frac{1}{2}$ or we repeat the argument.

Thus, we can apply Theorem 3 with $g$ being an eigenfunction of the operator $T$, taking into account that from Theorem 4 we know that, at least, $g \in H^{\frac{1}{2}-\epsilon}\left(\Gamma_{3}\right)$ for all $\epsilon>0$ (indeed, $g$ is probably more regular but we observe that we can only take $s<\frac{1}{2}$ in Theorem 3 ). Then we have the next result.

Corollary 4 There exists a positive constant $C$, depending on $\alpha$, such that for any eigenfunction $g$ of $T$

$$
\left\|T g-T_{h} g\right\|_{L^{2}\left(\Gamma_{3}\right)} \leq C\left(h \sqrt{\log \frac{1}{h}}\right)^{\frac{\sigma}{2}+\frac{1}{2}-\epsilon}\|g\|_{H^{\frac{1}{2}-\epsilon}\left(\Gamma_{3}\right)} .
$$

Now, recalling that since $1<\alpha<2, T$ is compact (see Proposition 2) we are able to apply the spectral approximation theory for compact operators given in [10]. We remark that since the linear operator $T$ on the Hilbert space $L^{2}\left(\Gamma_{3}\right)$ is self-adjoint, for each $\mu$ eigenvalue of $T$ the geometric and algebraic multiplicities of $\mu$ are equal.

Let $\mu$ be a fixed eigenvalue of $T$ with multiplicity $m$ and denote by $\mathcal{E}$ its eigenspace. From Lemma 1 we know that $T_{h}$ converge to $T$ in norm and so, there exist exactly $m$ eigenvalues of $T_{h}, \mu_{h}^{1}, \ldots, \mu_{h}^{m}$ repeated according to their algebraic multiplicities converging to $\mu$. Let $\mathcal{E}_{h}$ be the direct sum of the associated eigenspaces. Define the gap $\bar{\delta}$ between two subspaces, $\mathcal{X}$ and $\mathcal{Y}$ of $L^{2}\left(\Gamma_{3}\right)$

$$
\bar{\delta}(\mathcal{X}, \mathcal{Y}):=\max \{\delta(\mathcal{X}, \mathcal{Y}), \delta(\mathcal{Y}, \mathcal{X})\}
$$

with

$$
\delta(\mathcal{X}, \mathcal{Y})=\sup _{v \in \mathcal{X},\|v\|_{L^{2}\left(\Gamma_{3}\right)}=1}\left(\inf _{w \in \mathcal{Y}}\|v-w\|_{L^{2}\left(\Gamma_{3}\right)}\right)
$$

In view of Corollary 4, and [23, Theorem 1] we have the following result.

Lemma 2 There exists a constant $C$, dependent on $\alpha$ and the eigenvalue $\mu$ but independent on $h$, such that

$$
\bar{\delta}\left(\mathcal{E}, \mathcal{E}_{h}\right) \leq C\left(h \sqrt{\log \frac{1}{h}}\right)^{\frac{\sigma}{2}+\frac{1}{2}-\epsilon}
$$

Furthermore, from Corollary 4 and [10, Theorem 7.4] we also have the next Corollary, which for simplicity we state just for simple eigenvalues (see, for example, [10] for the general case). 
Corollary 5 Let $\mu$ be a simple eigenvalue of $T$ and let $\mu_{h}$ be the associated eigenvalue of $T_{h}$. Then, the corresponding eigenfunctions $u$ and $u_{h}$ can be chosen such that $\|u\|_{L^{2}\left(\Gamma_{3}\right)}=1,\left\|u_{h}\right\|_{L^{2}\left(\Gamma_{3}\right)}=1$ and

$$
\left\|u-u_{h}\right\|_{L^{2}\left(\Gamma_{3}\right)} \leq C\left(h \sqrt{\log \frac{1}{h}}\right)^{\frac{\sigma}{2}+\frac{1}{2}-\epsilon}
$$

with $C$ a strictly positive constant, dependent on $\alpha$ and the eigenvalue $\mu$ but independent on $h$.

Proof. Let $\mathcal{E}$ be set of (generalized) eigenfunctions for $\mu$. Then $\mathcal{E}$ is a one-dimensional subspace. Let $g \in \mathcal{E}$ be an eigenfunction with $\|g\|_{L^{2}\left(\Gamma_{3}\right)}=1$. Then

$$
\left\|\left.\left(T-T_{h}\right)\right|_{\mathcal{E}}\right\|_{\mathcal{L}\left(L^{2}\left(\Gamma_{3}\right)\right)}=\left\|T g-T_{h} g\right\|_{L^{2}\left(\Gamma_{3}\right)}
$$

and from Corollary 4 we obtain

$$
\left\|\left.\left(T-T_{h}\right)\right|_{\mathcal{E}}\right\|_{\mathcal{L}\left(L^{2}\left(\Gamma_{3}\right)\right)} \leq C\left(h \sqrt{\log \frac{1}{h}}\right)^{\frac{\sigma}{2}+\frac{1}{2}-\epsilon}\|g\|_{H^{\frac{1}{2}-\epsilon}\left(\Gamma_{3}\right)} .
$$

Now, from Lemma 4 (and $\alpha<2$ ) we know that $g$ is at least a function in $H^{\frac{1}{2}}\left(\Gamma_{3}\right.$ ), so we can write the last inequality as

$$
\left\|\left.\left(T-T_{h}\right)\right|_{\mathcal{E}}\right\|_{\mathcal{L}\left(L^{2}\left(\Gamma_{3}\right)\right)} \leq C\left(h \sqrt{\log \frac{1}{h}}\right)^{\frac{\sigma}{2}+\frac{1}{2}-\epsilon},
$$

where the fractional norm of $g$ was incorporated to $C$, the constant therefore depends only on the eigenvalue $\mu$ and is independent of $\epsilon$.

It is stated in [10, Theorem 7.4] that the eigenfunctions $u$ and $u_{h}$ in the statement of the Corollary can be chosen such that

$$
\left\|u-u_{h}\right\|_{L^{2}\left(\Gamma_{3}\right)} \leq C\left\|\left.\left(T-T_{h}\right)\right|_{\mathcal{E}}\right\|_{\mathcal{L}\left(L^{2}\left(\Gamma_{3}\right)\right)},
$$

from where we obtain the result.

Hence, with [10, Theorem 7.3] in mind, the convergence order for the approximate eigenvalues can be improved by using the following lemma.

Lemma 3 For all $\epsilon>0$ there exists a positive constant $C$ such that for any $f, g \in \mathcal{E}$

$$
\left|\int_{\Gamma_{3}}\left(T-T_{h}\right) f g\right| \leq C\left(h \sqrt{\log \frac{1}{h}}\right)^{1-\epsilon}\|f\|_{H^{\frac{1}{2}-\epsilon}\left(\Gamma_{3}\right)}\|g\|_{H^{\frac{1}{2}-\epsilon}\left(\Gamma_{3}\right)} .
$$

Proof. The proof follows the same ideas given in [2, Lemma 4.5], we include it for the sake of completeness. Let $f, g \in \mathcal{E}$ and let

$$
\begin{aligned}
& u=B f, \quad u_{h}=B_{h} f, \\
& v=B g, \quad v_{h}=B_{h} g .
\end{aligned}
$$

Then, we have that

$$
\int_{\Gamma_{3}}\left(T-T_{h}\right) f g=\int_{\Gamma_{3}}\left(u-u_{h}\right) g=\int_{\Gamma_{3}} u g-\int_{\Gamma_{3}} u_{h} g .
$$

On the other hand, from (3) and (5), we have that

$$
\begin{aligned}
& \int_{\Omega} \nabla u \nabla w=\int_{\Gamma_{3}} f w \quad \forall w \in V \\
& \int_{\Omega} \nabla v \nabla w=\int_{\Gamma_{3}} g w \quad \forall w \in V
\end{aligned}
$$


and

$$
\begin{array}{cl}
\int_{\Omega_{h}} \nabla u_{h} \nabla w_{h} & =\int_{\Gamma_{3}} f w_{h} \quad \forall w_{h} \in V_{h}, \\
\int_{\Omega_{h}} \nabla v_{h} \nabla w_{h} & =\int_{\Gamma_{3}} g w_{h} \quad \forall w_{h} \in V_{h} .
\end{array}
$$

In particular,

$$
\begin{gathered}
\int_{\Gamma_{3}} u g=\int_{\Omega} \nabla u \nabla v \\
\int_{\Gamma_{3}} u_{h} g=\int_{\Omega_{h}} \nabla v_{h} \nabla u_{h}
\end{gathered}
$$

and, since functions in $V_{h}$ restricted to $\Omega$ belong to $V$, we also have that

and

$$
\int_{\Omega_{h}} \nabla u_{h} \nabla v_{h}=\int_{\Gamma_{3}} f v_{h}=\int_{\Omega} \nabla u \nabla v_{h}
$$

$$
\int_{\Omega_{h}} \nabla v_{h} \nabla u_{h}=\int_{\Gamma_{3}} g u_{h}=\int_{\Omega} \nabla v \nabla u_{h} .
$$

As a consequence,

$$
\begin{aligned}
\int_{\Gamma_{3}} u g-\int_{\Gamma_{3}} u_{h} g & =\int_{\Omega} \nabla v \nabla u-\int_{\Omega} \nabla v \nabla u_{h}+\int_{\Omega_{h}} \nabla v_{h} \nabla u_{h}-\int_{\Omega} \nabla v_{h} \nabla u \\
& =\int_{\Omega} \nabla\left(u-u_{h}\right) \nabla\left(v-v_{h}\right)+\int_{\Omega_{h} \backslash \Omega} \nabla u_{h} \nabla v_{h} .
\end{aligned}
$$

Now, since $f$ and $g$ are eigenfunctions, $f, g \in H^{\frac{1}{2}-\epsilon}\left(\Gamma_{3}\right)$, and the solutions $u$ and $v$ are in $H^{\frac{3}{2}-\epsilon}(\Omega)$. Then, from (28) we can affirm that $\left\|u-u_{h}\right\|_{H^{1}(\Omega)} \leq C\left(h \sqrt{\log \frac{1}{h}}\right)^{\frac{1}{2}-\epsilon}\|f\|_{H^{\frac{1}{2}-\epsilon}\left(\Gamma_{3}\right)}$, and from (29) that $\left\|\nabla u_{h}\right\|_{L^{2}\left(\Omega_{h} \backslash \Omega\right)} \leq C\left(h \sqrt{\log \frac{1}{h}}\right)^{\frac{1}{2}-\epsilon}\|f\|_{H^{\frac{1}{2}-\epsilon}\left(\Gamma_{3}\right)}$ (analogously for the error $\left\|v-v_{h}\right\|_{H^{1}(\Omega)}$ and $\left.\left\|\nabla v_{h}\right\|_{L^{2}\left(\Omega_{h} \backslash \Omega\right)}\right)$ and the proof concludes.

Theorem 5 Let $\mu$ be a simple eigenvalue of $T$ and $\mu_{h}$ the associated eigenvalue of $T_{h}$. Then, for any $\epsilon>0$ there exists a constant $C$ such that

$$
\left|\mu-\mu_{h}\right| \leq C\left(h \sqrt{\log \frac{1}{h}}\right)^{1-\epsilon}
$$

Proof. It follows from the previous lemma, Corollary 4, and [10, Theorem 7.3].

We recall from Section 2 that problem (1) has a divergence sequence of positive eigenvalues with $\lambda$ being an eigenvalue of (1) if and only if $\lambda=\frac{1}{\mu}$ for an eigenvalue $\mu$ of $T$. Analogously, $\lambda_{h}$ is an eigenvalue of (2) if and only if $\lambda_{h}=\frac{1}{\mu_{h}}$ with $\mu_{h}$ being an eigenvalue of $T_{h}$. The boundedness of eigenvalues of $T$ and $T_{h}$ gives us the next Corollary.

Corollary 6 Let $\lambda$ be a simple eigenvalue of (1). Then, for any h small enough, there exists an eigenvalue $\lambda_{h}$ of (2) such that $\lambda_{h} \rightarrow \lambda$ when $h \rightarrow 0$ and we have, for any $\epsilon>0$,

$$
\left|\lambda-\lambda_{h}\right| \leq C\left(h \sqrt{\log \frac{1}{h}}\right)^{1-\epsilon}
$$

with the constant $C$ independent of $h$ (but may depend of $\epsilon$ ). 
The next lemma gives a relationship between the eigenvalue $\lambda$ of problem (1) and its approximation $\lambda_{h}$ of problem (2) and it is, in particular, a tool to obtain error estimates for eigenfunctions in $H^{1}$ norm.

Lemma 4 Let $(\lambda, u)$ and $\left(\lambda_{h}, u_{h}\right)$ be eigenpairs solutions of problems (1) and (2) respectively, with $\|u\|_{L^{2}(\Omega)}=1$ and $\left\|u_{h}\right\|_{L^{2}(\Omega)}=1$. Then we have that

$$
\lambda_{h}-\lambda=\left\|\nabla\left(u_{h}-u\right)\right\|_{L^{2}(\Omega)}^{2}-\lambda\left\|u_{h}-u\right\|_{L^{2}\left(\Gamma_{3}\right)}^{2}+\left\|\nabla u_{h}\right\|_{L^{2}\left(\Omega_{h} \backslash \Omega\right)}^{2} .
$$

Proof. The proof follows the same ideas of, for example, [10, Lemma 9.1], we include it for the sake of completeness. We have

$$
\begin{aligned}
\lambda+\lambda_{h} & =\lambda\|u\|_{L^{2}\left(\Gamma_{3}\right)}^{2}+\lambda_{h}\left\|u_{h}\right\|_{L^{2}\left(\Gamma_{3}\right)}^{2}=a(u, u)+a_{h}\left(u_{h}, u_{h}\right) \\
& =a\left(u-u_{h}, u-u_{h}\right)+2 a\left(u, u_{h}\right)+\left\|\nabla u_{h}\right\|_{L^{2}\left(\Omega_{h} \backslash \Omega\right)}^{2} \\
& =\left\|\nabla\left(u-u_{h}\right)\right\|_{L^{2}(\Omega)}^{2}+2 \lambda \int_{\Gamma_{3}} u u_{h}+\left\|\nabla u_{h}\right\|_{L^{2}\left(\Omega_{h} \backslash \Omega\right)}^{2} \\
& =\left\|\nabla\left(u-u_{h}\right)\right\|_{L^{2}(\Omega)}^{2}+2 \lambda-\lambda\left\|u-u_{h}\right\|_{L^{2}\left(\Gamma_{3}\right)}^{2}+\left\|\nabla u_{h}\right\|_{L^{2}\left(\Omega_{h} \backslash \Omega\right)}^{2},
\end{aligned}
$$

then (33) holds.

Theorem 6 Let $\lambda$ be a (simple) eigenvalue of problem (1) and let $\lambda_{h}$ be the associated eigenvalue solution of (2). Then the corresponding eigenfunctions $u$ and $u_{h}$ can be chosen such that $\|u\|_{L^{2}\left(\Gamma_{3}\right)}=\left\|u_{h}\right\|_{L^{2}\left(\Gamma_{3}\right)}=$ 1 and

$$
\left\|u-u_{h}\right\|_{H^{1}(\Omega)} \leq C\left(h \sqrt{\log \frac{1}{h}}\right)^{\frac{1}{2}-\epsilon}
$$

with $C$ independent of $h$.

Proof. From (33) we have

$$
\left\|\nabla\left(u-u_{h}\right)\right\|_{L^{2}(\Omega)}^{2}+\left\|\nabla u_{h}\right\|_{L^{2}\left(\Omega_{h} \backslash \Omega\right)}^{2}=\lambda_{h}-\lambda+\lambda\left\|u-u_{h}\right\|_{L^{2}\left(\Gamma_{3}\right)}^{2}
$$

and therefore

$$
\left\|\nabla\left(u-u_{h}\right)\right\|_{L^{2}(\Omega)} \leq\left|\lambda_{h}-\lambda\right|+\lambda\left\|u-u_{h}\right\|_{L^{2}\left(\Gamma_{3}\right)}^{2} .
$$

Then Corollary 5 and Corollary 6 give us

$$
\left\|\nabla\left(u-u_{h}\right)\right\|_{L^{2}(\Omega)}^{2} \leq C\left\{\left(h \sqrt{\log \frac{1}{h}}\right)^{1-\epsilon}+|\lambda|\left(h \sqrt{\log \frac{1}{h}}\right)^{1+\sigma-\epsilon}\right\} \leq C\left(h \sqrt{\log \frac{1}{h}}\right)^{1-\epsilon}
$$

as we wanted, with the constant $C$ depending on $\lambda$ and $\alpha$, but independent of $h$.

\section{Numerical aspects and numerical experiments}

In this section we analyze numerical aspects concerning the solution of the discrete problem (2), we present numerical approximations of the first eigenvalue and the numerical orders of convergence. 


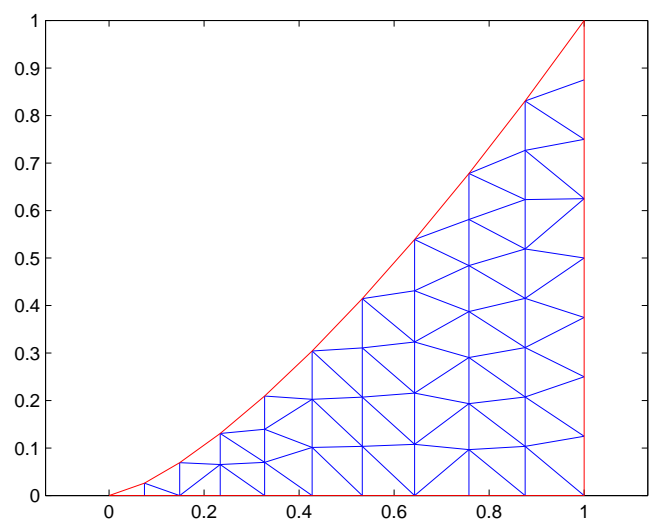

Fig. 4 Graded mesh with $\alpha=1.4$ and $n=10$.

\subsection{Numerical aspects}

First, we show that meshes satisfying the hypotheses M.1 - M.6 and (Ha) can be constructed. In fact, to define the mesh $\mathcal{T}_{h}$, with $h=1 / n$ we recall the method given in [17, page 393] and [24] which has been also used in $[1,2,4]$.

1. Introduce the partition of the interval $(0,1)$ given by

$$
x_{j}=\left(\frac{j}{n}\right)^{\frac{2}{3-\alpha}} \quad 0 \leq j \leq n .
$$

2. Take the points $\left(x_{j}, 0\right)$ in $\Gamma_{1},\left(x_{j}, x_{j}^{\alpha}\right)$ in $\Gamma_{3}$, and for $j>1$, divide each of the vertical lines $\left\{\left(x_{j}, y\right)\right.$ : $\left.0 \leq y \leq x_{j}^{\alpha}\right\}$ uniformly into subintervals such that each has length $\sim x_{j}-x_{j-1}$.

Figure 4 shows an example of one of these meshes.

Now, we analyze some aspects about the resolution of the generalized eigenvalue problem (2).

Let $\mathcal{N}_{h, N D}$ be the set of vertices of the mesh excluding the ones on the Dirichlet boundary $\Gamma_{2}$, and split it as $\mathcal{N}_{h, N D}=\mathcal{N}_{h, o} \cup \mathcal{N}_{h, 3}$ with $\mathcal{N}_{h, 3}$ being the subset of $\mathcal{N}_{h, N D}$ consisting of the vertices which belong to a triangle having two vertices on $\Gamma_{3}$, and $\mathcal{N}_{h, o}=\mathcal{N}_{h, N D} \backslash \mathcal{N}_{h, 3}$.

We introduce the matrices

$$
\boldsymbol{A}_{r s}:=\left(\int_{\Omega} \nabla \beta_{i} \cdot \nabla \beta_{j}\right)_{P_{i} \in \mathcal{N}_{h, r}, P_{j} \in \mathcal{N}_{h, s}}, \quad \boldsymbol{B}_{r s}:=\left(\int_{\Gamma_{3}} \beta_{i} \beta_{j}\right)_{P_{i} \in \mathcal{N}_{h, r}, P_{j} \in \mathcal{N}_{h, s}} \quad r, s=o, 3,
$$

with $\left\{\beta_{i}\right\}_{P_{i} \in \mathcal{N}_{h, N D}}$ being the nodal basis, that is, $\beta_{i} \in V_{h}$ with $\beta_{i}\left(P_{j}\right)=\delta_{i j}$. Clearly we have

$$
\boldsymbol{B}_{o o}=\mathbf{0}, \quad \boldsymbol{B}_{o 3}=\boldsymbol{B}_{3 o}^{t}=\mathbf{0} .
$$

A function $v \in V_{h}$ can be written as

$$
v=\sum_{P_{i} \in \mathcal{N}_{h, o}} v\left(P_{i}\right) \beta_{i}+\sum_{P_{j} \in \mathcal{N}_{h, 3}} v\left(P_{j}\right) \beta_{j}
$$

Introducing the vectors

$$
\boldsymbol{u}_{o}:=\left(u_{h}\left(P_{i}\right)\right)_{P_{i} \in \mathcal{N}_{h, o}} \in \mathbb{R}^{N_{o}} \quad \text { and } \quad \boldsymbol{u}_{3}:=\left(u_{h}\left(P_{i}\right)\right)_{P_{i} \in \mathcal{N}_{h, 3}} \in \mathbb{R}^{\mathrm{n}_{3}},
$$


with $n_{i}=\# \mathcal{N}_{h, i}, i=o, 3$, we have that $u_{h}$ is defined by the system

$$
\left(\begin{array}{ll}
\boldsymbol{A}_{o o} & \boldsymbol{A}_{o 3} \\
\boldsymbol{A}_{o 3}^{\prime} & \boldsymbol{A}_{33}
\end{array}\right)\left(\begin{array}{l}
\boldsymbol{u}_{o} \\
\boldsymbol{u}_{3}
\end{array}\right)=\lambda_{h}\left(\begin{array}{cc}
\mathbf{0} & \mathbf{0} \\
\mathbf{0} & \boldsymbol{B}_{33}
\end{array}\right)\left(\begin{array}{l}
\boldsymbol{u}_{o} \\
\boldsymbol{u}_{3}
\end{array}\right)
$$

This system can be transformed into a standard symmetric generalized eigenvalue problem. We observe that the matrix on the left hand side of (35) is symmetric and positive definite. Then, since the submatrix $\boldsymbol{A}_{o o}$ is invertible (indeed, symmetric and positive definite, too), we can write

$$
\boldsymbol{u}_{o}=-\boldsymbol{A}_{o o}^{-1} \boldsymbol{A}_{o 3} \boldsymbol{u}_{3} .
$$

Now, by eliminating $\boldsymbol{u}_{o}$ from (35) we arrive at

$$
\boldsymbol{C} \boldsymbol{u}_{3}=\lambda_{h} \boldsymbol{B}_{33} \boldsymbol{u}_{3},
$$

with $\boldsymbol{C}:=\boldsymbol{A}_{33}-\boldsymbol{A}_{o 3}^{t} \boldsymbol{A}_{o o}^{-1} \boldsymbol{A}_{o 3}$. This problem is equivalent to (35). Notice that although the matrix $\boldsymbol{C}$ is not sparse, its dimension is $\mathrm{n}_{3} \times \mathrm{n}_{3}$ and, hence significantly smaller than the size of system (35). Moreover, in actual computations, the matrix $\boldsymbol{A}_{o o}^{-1}$ is not explicitly computed, in fact, the columns of matrix $\boldsymbol{A}_{\text {oo }}^{-1} \boldsymbol{A}_{o 3}$ are obtained as the solution of linear systems with the same matrix $\boldsymbol{A}_{\text {oo }} \in \mathbb{R}^{N_{o} \times N_{o}}$, which is sparse, symmetric and positive definite. Then, this is a well posed (and very small) problem that can be efficiently solved by any standard eigensolver.

\subsection{Numerical experiments}

In Table 1 we present numerical approximations of the first six eigenvalues of the Steklov problem (2) for $\alpha=1.4$. Then, we solve the problem for different values of $\alpha$ with the purpose of try to estimate the order of the approximation.

\begin{tabular}{|c|c|c|c|c|c|c|c|}
\hline$h$ & \# nodes & $\lambda_{h, 1}$ & $\lambda_{h, 2}$ & $\lambda_{h, 3}$ & $\lambda_{h, 4}$ & $\lambda_{h, 5}$ & $\lambda_{h, 6}$ \\
\hline $2^{-3}$ & 53 & 0.8041273434 & 2.9242421362 & 5.2402919893 & 7.9437328154 & 11.1409802166 & 15.0438670074 \\
$2^{-4}$ & 162 & 0.7946744205 & 2.7816190225 & 4.8508461523 & 7.0132049915 & 9.3718201883 & 11.9190288842 \\
$2^{-5}$ & 571 & 0.7916544790 & 2.7286690230 & 4.7131025238 & 6.7138702509 & 8.8188973607 & 10.9909317572 \\
$2^{-6}$ & 2157 & 0.7907200884 & 2.7095889679 & 4.6605475331 & 6.6028687328 & 8.6288870966 & 10.6936643446 \\
$2^{-7}$ & 8382 & 0.7904487012 & 2.7035314255 & 4.6428771968 & 6.5641325567 & 8.5619389967 & 10.5898209679 \\
$2^{-8}$ & 33102 & 0.7903710292 & 2.7016789808 & 4.6371925732 & 6.5511662493 & 8.5388907520 & 10.5534888493 \\
\hline
\end{tabular}

Table 1 Numerical approximation of the first six eigenvalues for $\alpha=1.4$ with graded meshes.

Assuming that the error $\left|\lambda-\lambda_{h}\right|$ behaves as $C(h \log h)^{R}$ (with $h$ being the parameter defining the graded meshes, which behaves as $\frac{1}{\sqrt{N}}$, where $N$ is the number of nodes), Table 2 presents the experimental order of convergence (e.o.c), $R=R(\alpha)$, which, for different values of $\alpha$ is computed by using a leastsquares fitting with the last four results of an iterative process, in which the last iteration is performed with $h=\frac{1}{256}$ for $\alpha \leq 1.5$, with $h=\frac{1}{128}$ for $\alpha=1.6$ and with $h=\frac{1}{32}$ for $\alpha \geq 1.7$ (we notice that, from (34), for a given value of $n, x_{1} \sim \frac{1}{n}$ when $\alpha$ is next to 1 while $x_{1} \sim \frac{1}{n^{2}}$ for $\alpha$ next to 2 and therefore the number of nodes increases considerably as $\alpha$ increases).

We observe that the e.o.c. increase when $\alpha$ approach 1, while when $\alpha$ approach 2 the order predicted by the theory seems to be obtained. We recall that, in view of Theorem 3, our estimates are restricted to the worst case in which the eigenfunctions are in $H^{\frac{1}{2}-\epsilon}$ for all $\epsilon>0$ (although they are probably more regular). 


\begin{tabular}{|c|c|c|c|c|c|c|}
\hline$\alpha$ & $\lambda_{h, 1}$ & $\lambda_{h, 2}$ & $\lambda_{h, 3}$ & $\lambda_{h, 4}$ & $\lambda_{h, 5}$ & $\lambda_{h, 6}$ \\
\hline 1.1 & 1.96 & 1.93 & 1.96 & 1.97 & 1.97 & 1.97 \\
1.2 & 1.92 & 1.88 & 1.91 & 1.93 & 1.94 & 1.96 \\
1.3 & 1.86 & 1.78 & 1.78 & 1.78 & 1.79 & 1.81 \\
1.4 & 1.79 & 1.78 & 1.60 & 1.54 & 1.52 & 1.51 \\
1.5 & 1.76 & 1.55 & 1.40 & 1.31 & 1.27 & 1.26 \\
1.6 & 1.62 & 1.31 & 1.04 & 0.97 & 0.94 & 0.99 \\
1.7 & 1.44 & 1.09 & 0.83 & 0.86 & 0.96 & 1.04 \\
1.8 & 1.33 & 1.06 & 0.90 & 1.11 & 1.47 & 1.92 \\
1.9 & 1.03 & 0.97 & 0.85 & 0.83 & 1.00 & 1.27 \\
\hline
\end{tabular}

Table 2 For the first six eigenvalues, the table shows the e.o.c with respect to the parameter $h$ for different values of $\alpha$ using graded meshes.

In all the cases computed we observe that the discrete sequence monotonically decreases with $h$. The reason for this behaviour could be that, from Lemma 4, we have

$$
\lambda_{h}-\lambda=\frac{\left(\left\|\nabla\left(u-u_{h}\right)\right\|_{L^{2}(\Omega)}^{2}+\left\|\nabla u_{h}\right\|_{L^{2}\left(\Omega_{h} \backslash \Omega\right)}^{2}\right)-\lambda_{h}\left\|u-u_{h}\right\|_{L^{2}\left(\Gamma_{3}\right)}^{2}}{1-\left\|u-u_{h}\right\|_{L^{2}\left(\Gamma_{3}\right)}^{2}},
$$

and for $h$ small the sign of $\lambda_{h}-\lambda$ depends on the difference of the two terms given in the numerator. Since $1<\alpha<2$ (and therefore $\sigma>0$ ), taking into account the results obtained in Corollary 5 , Theorem 6 and inequality (29), we would expect that the second one to be of higher order than the first one, we can conjecture that $\lambda_{h}-\lambda>0$ for $h$ small enough.

On the other hand, assuming $\lambda_{h}-\lambda>0$ and considering $\lambda_{H}$ and $\lambda_{h}$, eigenvalues of problem (2) with $H>h$, we can write $\lambda_{H}-\lambda_{h}=\lambda_{H}-\lambda-\left(\lambda_{h}-\lambda\right)$. Then, Theorem 5 would imply the monotonicity of the discrete sequence of eigenvalues.

We end this paper by highlighting that, the trace results introduced in Section 1 and the interpolation theorems obtained in Section 4 for cuspidal domains (joint with the theory developed in [1-4]) could be a fundamental tool to approximate another kind of boundary eigenvalue problems as, for example, the fluid-solid vibrations problems considered in [8].

Acknowledgements This work was partially supported by ANPCyT under grant PICT 2014-1771 and by CONICET under grant PIP (2014-2016) 11220130100184CO. MGA was also supported by Universidad de Buenos Aires under grant 20020130100205BA. ALL was also supported by Universidad de Buenos Aires under grant 20020120100050BA and by Universidad Nacional de Rosario under grant ING568.

\section{References}

1. Gabriel Acosta and María G. Armentano, Finite element approximations in a non-Lipschitz domain: Part II, Mathematics of Computation, 80 (276), 1949-1978 (2011).

2. Gabriel Acosta and María G. Armentano, Eigenvalue problems in a non-Lipschitz domain, IMA Journal of Numerical Analysis, 34 (1), 83-95 (2014).

3. G. Acosta, M. G. Armentano, R. G. Durán, and A. L. Lombardi, Nonhomogeneous Neumann problem for the Poisson equation in domains with an external cusp, J. Math. Anal. Appl., 310 397-411 (2005).

4. G. Acosta, M. G. Armentano, R. G. Durán and A. L. Lombardi, Finite element approximations in a non-Lipschitz domain, SIAM J. Numer. Anal. 45(1), 277-295 (2007).

5. R. A. Adams, Sobolev Spaces, Academic Press, New York, (1975).

6. A. B. Andreev and T. D. Todorov Isoparametric finie-element approximation of a Steklov eigenvalue problem, IMA Journal of Numerical Analysis, 24, 309-322, (2004).

7. M. G. Armentano, The effect of reduced integration in the Steklov eigenvalue problem, Math. Mod. and Numer. Anal. $\left(\mathrm{M}^{2} \mathrm{AN}\right), \mathbf{3 8}(1), 27-36(2004)$.

8. M. G. Armentano, C. Padra, R. Rodriguez and M. Scheble, An hp finite element adaptive scheme to solve the Laplace model for fluid- solid vibrations , Computer Methods in Applied Mechanics and Engineering, 200, (2011) 178-188.

9. M. G. Armentano and C. Padra, A posteriori error estimates for the Steklov eigenvalue problem, Applied Numerical Mathematics, 58(5) 593-601, (2008). 
10. I. Babuška and J. Osborn, Eigenvalue Problems, Handbook of Numerical Analysis, vol. II, Finite Element Methods (Part.1), (1991).

11. A. Bermúdez, R. Rodríguez and D. Santamarina, A finite element solution of an added mass formulation for coupled fluid-solid vibrations, Numerische Mathematik, 87, 201-227, (2000).

12. D. Boffi, Finite element aproximation of eigenvalue problems, Acta Numerica 19, 1-120 (2010).

13. K. Brewster and M. Mitrea, Boundary value problems in weighted Sobolev spaces on Lipschitz manifolds, Memoirs Diff. Eq. Math. Phys. 60, 15-55 (2013).

14. J. Canavati and A. Minsoni A discontinuous Steklov problem with an application to water waves, J. Math. Anal. Appl. 69, 540-558 (1979).

15. T. Dupont, R. Scott, Polynomial approximations of functions in Sobolev Spaces, Math. Comp. 34 , 441-463 (1980).

16. A. Girouard, I. Polterovich, Spectral geometry of the Steklov problem, J. Spectr. Theory 7, 321-359 (2017).

17. P. Grisvard, Elliptic Problems in Nonsmooth Domain, Pitman Boston, 1985.

18. D. Kim, Elliptic equations with nonzero boundary conditions in weighted Sobolev spaces, J. Math. Anal. Appl. 337, 1465-1479 (2008).

19. V. Kozlov, N. Kuznetsov, O. Motygin, On the two-dimensional sloshing problem, Proc. R. Soc. Lond. A 460, 2587-2603 (2004).

20. J.L. Lions, E. Magenes, Problémes aux limites non homogénes et applications. vol 1. Dunod. Paris (1968).

21. V.G. Maz'ya, S.V. Poborchi, Differentiable functions on bad domains. World Scientific. Singapore (1997).

22. S. A. Nazarov, J. Sokolowski and J. Taskinen, Neumann Laplacian on a domain with tangential components in the boundary, Ann. Acad. Sci. Fenn. Math. 34 (1), 131-143, (2009).

23. J. Osborn, Spectral Approximation for Compact Operators, Math. Comp. 29, 712-725 (1975).

24. G. Raugel, Résolution numérique par une méthode d'éléments finis du problème Dirichlet pour le laplacien dans un polygone, C. R. Acad. Sci. Paris Ser. A, 286, A791-A794 (1978).

25. L. R. Scott, S. Zhang, Finite element interpolation of nonsmooth functions satisfying boundary conditions. Math. Comp. 54, 483-493 (1990).

26. H. F. Weinberger, Variational Methods for Eigenvalue Approximation, SIAM, Philadelphia, (1974). 